\title{
Article
}

\section{Spatial Navigation and Visuospatial Strategies in Typical and Atypical Aging}

\author{
Martina Laczó ${ }^{1,2}$, Jan M. Wiener ${ }^{3}$, Jana Kalinova ${ }^{1}$, Veronika Matuskova ${ }^{1,2}$, Martin Vyhnalek ${ }^{1,2}$, Jakub Hort ${ }^{1,2}$ \\ and Jan Laczó ${ }^{1,2, *}$ \\ 1 Memory Clinic, Department of Neurology, Charles University, Second Faculty of Medicine, Motol University \\ Hospital, 15006 Prague, Czech Republic; martina.laczo@gmail.com (M.L.); kalinja@email.cz (J.K.); \\ veronika.matuskova@lfmotol.cuni.cz (V.M.); martin.vyhnalek@lfmotol.cuni.cz (M.V.); \\ jakub.hort@lfmotol.cuni.cz (J.H.) \\ 2 International Clinical Research Center, St. Anne's University Hospital Brno, 65691 Brno, Czech Republic \\ 3 Department of Psychology, Ageing and Dementia Research Centre, Bournemouth University, \\ Poole BH12 5BB, UK; jwiener@bournemouth.ac.uk \\ * Correspondence: jan.laczo@lfmotol.cuni.cz
}

Citation: Laczó, M.; Wiener, J.M.; Kalinova, J.; Matuskova, V.; Vyhnalek, M.; Hort, J.; Laczó, J. Spatial Navigation and Visuospatial Strategies in Typical and Atypical Aging. Brain Sci. 2021, 11, 1421. https://doi.org/10.3390/brainsci 11111421

Academic Editor: Tina Iachini

Received: 15 September 2021

Accepted: 23 October 2021

Published: 27 October 2021

Publisher's Note: MDPI stays neutral with regard to jurisdictional claims in published maps and institutional affiliations.

Copyright: (C) 2021 by the authors. Licensee MDPI, Basel, Switzerland. This article is an open access article distributed under the terms and conditions of the Creative Commons Attribution (CC BY) license (https:// creativecommons.org/licenses/by/ $4.0 /)$.

\begin{abstract}
Age-related spatial navigation decline is more pronounced in patients with mild cognitive impairment (MCI) and Alzheimer's disease (AD) dementia. We used a realistic-looking virtual navigation test suite to analyze different aspects of visuospatial processing in typical and atypical aging. A total of 219 older adults were recruited from the Czech Brain Aging Study cohort. Cognitively normal older adults $(\mathrm{CN} ; n=78)$, patients with amnestic MCI $(n=75)$, and those with mild AD dementia $(n=66)$ underwent three navigational tasks, cognitive assessment, and brain MRI. Route learning and wayfinding/perspective-taking tasks distinguished the groups as performance and learning declined and specific visuospatial strategies were less utilized with increasing cognitive impairment. Increased perspective shift and utilization of non-specific strategies were associated with worse task performance across the groups. Primacy and recency effects were observed across the groups in the route learning and the wayfinding/perspective-taking task, respectively. In addition, a primacy effect was present in the wayfinding/perspective-taking task in the $\mathrm{CN}$ older adults. More effective spatial navigation was associated with better memory and executive functions. The results demonstrate that a realistic and ecologically valid spatial navigation test suite can reveal different aspects of visuospatial processing in typical and atypical aging.
\end{abstract}

Keywords: mild cognitive impairment; Alzheimer's disease; spatial navigation; route learning; wayfinding; perspective taking; navigation strategies; visuospatial functions

\section{Introduction}

Spatial navigation is a complex cognitive ability important in everyday life for moving in both familiar and unfamiliar environments. Successful spatial navigation requires a combination of different navigation strategies. One of them is route learning (i.e., response learning), which entails creating a sequence of body movements (e.g., remembering right, left, right) or creating a series of stimulus-response associations when remembering associations of direction change with a specific landmark (e.g., "Turn left at the shop") [1]. This strategy is referred to as egocentric navigation and is primarily based on self-motion perception but can also exploit environmental cues by creating self-to-object relations [2]. Route learning is primarily supported by the parietal cortex and the caudate nucleus [3].

A more flexible navigation strategy is wayfinding (i.e., place learning) where a navigator creates a mental representation (i.e., "cognitive map") of the environment based on landmarks that are used as orientation cues. This entails the recognition of object-to-object relationships, and this strategy is referred to as allocentric navigation [2]. Wayfinding is primarily supported by the hippocampus and related medial temporal lobe structures [4]. 
Route learning and wayfinding are supported by different brain regions and are used in parallel and complement each other when navigating in the environment [5]. While the environment is typically perceived and encoded from the navigator's perspective during navigation, successful navigation requires that the travelled route is recalled from a different direction, for example, when retracing a route (i.e., when navigating from the destination back to the starting place of a route). The flexibility to imagine scenes and environments from different viewpoints is referred to as perspective taking [2]. Perspective taking is supported by the parietal and temporal cortex [3].

Aging is associated with declines in spatial navigation abilities as documented by numerous studies [6,7]. Age-related spatial navigation declines are more pronounced in wayfinding than route learning, which is typically preserved for longer [6,8-10]. This decline could be a result of mild age-related functional and structural changes in the hippocampus and related medial temporal lobe structures [11,12]. Consequently, aging leads to an increasing preference for the route-learning strategy likely due to compensatory recruitment of extrahippocampal navigation strategies $[8,13]$.

Alzheimer's disease (AD) is characterized by profound spatial navigation impairment. This is likely to be a consequence of severe functional and structural changes in the brain regions that support spatial navigation, especially the hippocampus [14], the entorhinal cortex [15], and the parietal cortex [16], which are present in the early stages of the disease. $\mathrm{AD}$ is associated with route learning and wayfinding deficits. Wayfinding deficits in patients with mild cognitive impairment $(\mathrm{MCI})$ and mild dementia were described in realworld environments when navigating in a hospital setting [17] and an enclosed circular arena [18], and in virtual environments when navigating in a virtual hallway complex [19].

In $\mathrm{AD}$, wayfinding deficits are associated with smaller volumes of the hippocampus, entorhinal cortex, and basal forebrain [20-22]. Route-learning deficits are also present in $\mathrm{AD}$ [23] reflecting typical changes in the parietal cortex [24] and caudate nucleus [25]. Route-learning strategy preference increases over wayfinding with the progression of $\mathrm{AD}$ and reflects the recruitment of extrahippocampal navigation strategies [26]. In addition, the ability to imagine scenes during navigation from different spatial perspectives (viewpoint) (i.e., perspective taking) declines with age [27]. This deficit, which can be detected by virtual and paper-based tests, is more pronounced in AD [28].

In general, spatial navigation, especially wayfinding [6,8] and perspective taking [27], declines with aging. However, $\mathrm{AD}$ is associated with a greater spatial navigation decline including wayfinding, perspective taking, and route-learning, which is preserved in normal aging, and changes in preference of navigational strategies. These differences in profiles of spatial navigation dysfunctions indicate that spatial navigation assessment could be a useful tool to differentiate normal aging from the early stages of AD.

Visuospatial functions are important for all types of spatial navigation as visuospatial perception and processing allow the navigator to locate relevant stimuli or landmarks in the environment, to make spatial judgments, and to visually discriminate different scenes [29]. Visuospatial perception and spatial information processing decline in older age $[30,31]$. Patients with AD experience even greater disruption of visuospatial functions [32,33]. Specifically, AD is associated with altered scene exploration and reduced utilization of important visual landmarks in the environment, which can cause patients to overlook landmarks critical for navigation [34] and may thus contribute to spatial navigation impairments. Visuospatial processing may influence the selection of a navigation strategy because the strategy choice depends on the perception of visual information in the environment $[35,36]$. Specifically, in the route-learning task, participants prefer using visual landmarks rather than geometric information to navigate in the environment [35]. Furthermore, spatial navigation is more accurate in virtual environments that feature visual landmarks $[35,36]$. Collectively, these studies demonstrate that visual input is highly important for strategy selection and successful spatial navigation.

Spatial navigation assessment should be ideally performed using an ecologically valid test that is easy to explain and where participants navigate in a realistic-looking 
environment. When using realistic-looking environments, it is possible to mimic reallife navigation and thus provide the most accurate measure of everyday-life navigation abilities [37]. The Navigation Test Suite (https: / / osf.io / mx52y / , accessed on 16 March 2017) designed by [38] consists of three spatial tasks in a realistic-looking virtual city: A routerepetition task evaluating route learning, and route-retracing and directional-approach tasks evaluating wayfinding, which can be also solved using perspective taking. The Navigation Test Suite was previously used to assess the effects of normal aging on spatial navigation [38]. The young participants outperformed the older participants in all three tasks (i.e., route-repetition, route-retracing, and directional-approach tasks). Specifically, older participants' performance improved across the sessions in the route-repetition task but not in the route-retracing task. The young participants' performance, in contrast, improved in both tasks. In the directional-approach task, where participants had to recall the street from which they originally approached an intersection when approaching it from a viewpoint they had not experienced before, the older adults showed a greater decline in performance than the young participants when the perspective shift between encoding and retrieval was higher.

We built on previous research using the Navigation Test Suite [38] and aimed to (1) assess differences in spatial navigation performance between cognitively normal (CN) older adults and patients with amnestic MCI (aMCI) and mild AD dementia, (2) evaluate differences in navigation strategy selection and the effect of strategy selection on spatial navigation performance, (3) analyze learning across the experimental sessions and the effect of perspective shift on spatial navigation performance, and (4) analyze serial position performance, which refers to primacy and recency effects, which means that information perceived at the beginning (primacy effect) and at the end (recency effect) are remembered better than information in the middle of a route.

We hypothesized that: (1) the participants with aMCI and, even more, the participants with mild AD dementia would have less accurate spatial navigation performance in the route-learning, wayfinding, and perspective-taking task compared to the CN older adults; (2) the participants with aMCI and mild AD dementia would more often experience difficulties with creating an effective navigation strategy, which would lead to worse spatial navigation performance, (3) learning across the experimental sessions would be present in the $\mathrm{CN}$ group, less so in the aMCI group, and expressed the least in the mild AD dementia group, and (4) the serial position would be present differently in the $\mathrm{CN}$, aMCI and mild AD dementia groups.

The novelty of this study is based on the fact that it combines three different spatial navigation tasks (i.e., route-learning, route-retracing, and wayfinding/perspective taking) in one ecologically valid spatial navigation paradigm to comprehensively assess differences between typical and atypical aging. Next, our study brings new information about differences in the selection and use of navigation strategies in $\mathrm{CN}$ older adults and patients with aMCI and mild AD dementia and their associations with spatial navigation performance. Further, our study sheds new light on the serial position effect in spatial navigation. Up to now, the serial position effect has been assessed primarily in memory tests where a characteristic pattern of the serial position effect is preserved in older adults even when overall memory decreases [39], whereas patients with MCI typically show a primacy effect lower than the recency effect [40]. A recent study showed primacy and recency effects in route learning in young and older adults [41]. To the best of our knowledge, our study is the first to analyze primacy and recency effects in spatial navigation in patients with MCI and AD dementia. The Navigation Test Suite also allows us to assess the effect of spatial navigation learning and the effect of perspective shift on spatial navigation performance, which have been reported previously $[18,28]$ but not in an ecologically valid and realistic-looking large-scale virtual environment. 


\section{Methods}

\subsection{Participants}

A total of 219 participants were recruited from the Czech Brain Aging Study cohort [42] at the Memory Clinic of the Charles University, Second Faculty of Medicine and Motol University Hospital in Prague, Czech Republic, and signed an informed consent approved by the local ethics committee [43]. The participants with cognitive deficit were referred to the Memory Clinic by general practitioners and neurologists for memory complaints reported by themselves and/or by their relatives. $\mathrm{CN}$ older participants were recruited from the University of the Third Age, senior centers (e.g., the Elpida center), and relatives of the participants and hospital staff [44].

All participants underwent clinical and laboratory evaluations, comprehensive cognitive assessment, brain magnetic resonance imaging (MRI), and completed the Navigation Test Suite. The participants were classified into three groups: mild AD dementia, aMCI, and $\mathrm{CN}$ older adults based on clinical criteria, the information provided by the participants and their informants, and cognitive assessment. The demographic and cognitive characteristics are listed in Table 1.

Table 1. Characteristics of study participants.

\begin{tabular}{|c|c|c|c|c|c|}
\hline Variables & $\begin{array}{c}\mathrm{CN} \\
(n=78)\end{array}$ & $\begin{array}{c}\text { aMCI } \\
(n=75)\end{array}$ & $\begin{array}{l}\text { Mild AD Dementia } \\
\qquad(n=66)\end{array}$ & $p$ Values & Effect Sizes \\
\hline Age (years) & $68.22(6.80)$ & $71.49(7.38) *$ & $75.91(8.06)^{* * *++}$ & $<0.001$ & 0.15 \\
\hline Women, n (\%) & $56(72)$ & $33(44)$ & $42(64)$ & 0.002 & 0.24 \\
\hline Education (years) & $15.97(2.03)$ & $14.91(2.52) *$ & $14.29(3.01)^{* * *}$ & $<0.001$ & 0.07 \\
\hline MMSE (score) & $29.06(1.25)$ & $27.53(1.91)^{* * *}$ & $22.55(2.75)^{* * *+++}$ & $<0.001$ & 0.65 \\
\hline GDS-15 (score) & $2.04(2.25)$ & $2.49(2.29)$ & $2.90(2.61)$ & 0.130 & 0.02 \\
\hline BAI (score) & $7.46(6.58)$ & $7.41(6.81)$ & $7.56(6.44)$ & 0.993 & 0.00 \\
\hline RAVLT 1-5 (score) & $55.83(6.86)$ & $35.19(8.49)^{* * *}$ & $27.08(6.89)^{* * *+++}$ & $<0.001$ & 0.71 \\
\hline RAVLT 30 (score) & $11.97(1.91)$ & $4.29(2.92)^{* * *}$ & $2.29(3.75)^{* * *++}$ & $<0.001$ & 0.71 \\
\hline LM-IR (score) & $17.42(3.64)$ & $11.91(4.37)^{* * *}$ & $7.16(3.40)^{* * *+++}$ & $<0.001$ & 0.53 \\
\hline LM-DR (score) & $16.19(3.87)$ & $8.35(5.75)^{* * *}$ & $2.84(4.50)^{* * *+++}$ & $<0.001$ & 0.56 \\
\hline Stroop (seconds) & $28.34(8.10)$ & $38.69(15.78) * *$ & $59.87(29.82)^{* * *+++}$ & $<0.001$ & 0.31 \\
\hline TMT A (seconds) & $37.97(10.89)$ & $48.47(18.33)$ * & $72.60(41.00)^{* * *+++}$ & $<0.001$ & 0.24 \\
\hline TMT B (seconds) & $86.19(31.46)$ & $140.04(70.87)^{* * *}$ & $243.44(72.28) * * *+++$ & $<0.001$ & 0.52 \\
\hline COWAT (score) & $51.21(10.20)$ & $40.38(10.51)^{* * *}$ & $33.06(14.15) * * *++$ & $<0.001$ & 0.29 \\
\hline ROCFT-C (score) & $31.18(3.30)$ & $26.69(4.94)^{* * *}$ & $25.19(7.36)^{* * *}$ & $<0.001$ & 0.20 \\
\hline ROCFT-R (score) & $19.42(5.61)$ & $9.20(5.69)^{* * *}$ & $4.66(5.20)^{* * *}$ & $<0.001$ & 0.55 \\
\hline DSF (score) & $9.65(2.06)$ & $8.83(1.79)^{*}$ & $8.04(1.88)^{* * *}$ & $<0.001$ & 0.10 \\
\hline DSB (score) & $6.88(1.75)$ & $5.84(1.76)^{* * *}$ & $4.88(1.34)^{* * *++}$ & $<0.001$ & 0.19 \\
\hline CDT (score) & $15.19(1.13)$ & $14.28(1.78)^{* *}$ & $12.82(2.32) * * *+++$ & $<0.001$ & 0.22 \\
\hline SVF Animals (score) & $27.32(4.84)$ & $19.72(5.41)^{* * *}$ & $14.30(5.47)^{* * *+++}$ & $<0.001$ & 0.50 \\
\hline BNT (no. of errors) & $1.53(1.93)$ & $4.01(3.13)^{* * *}$ & $7.26(3.72)^{* * *+++}$ & $<0.001$ & 0.37 \\
\hline
\end{tabular}

Demographic and cognitive characteristics. Values are mean (SD) except for gender. $p$ Values refer to the main effect across all groups. For $p$ values indicating the level of significance compared to the $\mathrm{CN}$ group: ${ }^{*} p<0.05 ; * * p<0.01 ; * * * p<0.001$ and compared to the aMCI group: ${ }^{++} p<0.01 ;^{+++} p<0.001$. Effect sizes were calculated as Cramér's V for the $\chi^{2}$ test (gender) and partial eta-squared for one-way and mixed analyses of variance (all other variables). CN, cognitively normal; aMCI, amnestic mild cognitive impairment; mild AD dementia, mild Alzheimer's disease dementia; MMSE, Mini-Mental State Examination; GDS-15, Geriatric Depression Scale 15-item version; BAI, Beck Anxiety Inventory; RAVLT, Rey Auditory Verbal Learning Test; RAVLT 1-5, trials 1 to 5 total; RAVLT 30, delayed word recall after 30 min; LM-IR, Logical Memory—Immediate Recall; LM-DR, Logical Memory—Delayed Recall; Stroop, Prague Stroop Test—colors; TMT A and B, Trail Making Tests A and B; COWAT, Controlled Oral Word Association Test (Czech version with letters N, K and P); ROCFT-C, Rey-Osterrieth Complex Figure Test-the Copy condition; ROCFT-R, Rey-Osterrieth Complex Figure Test-the Recall condition after 3 min; DSF, Digit Span Forward total score; DSB, Digit Span Backward total score; CDT, Clock Drawing Test-Cohen's scoring; SVF, Semantic Verbal Fluency; BNT, Boston Naming Test.

(1) Participants with mild AD dementia $(n=66)$ met the clinical criteria for AD dementia [45] with evidence of progressive cognitive impairment in at least two cognitive domains including memory (i.e., score lower than 1.5 standard deviations (SDs) below the age- and education-adjusted norms in any memory test and in at least one other non-memory cognitive test) [26] and significant impairment in activities of daily 
living and had hippocampal atrophy (i.e., pathological medial temporal lobe atrophy score) [46].

(2) Participants with aMCI $(n=75)$ met the clinical criteria for aMCI [47] including memory complaints, evidence of memory impairment (i.e., score lower than 1.5 SDs below the age- and education-adjusted norms in any memory test) [26], generally intact activities of daily living, and the absence of dementia.

(3) CN participants $(n=78)$ did not report any cognitive complaints and had cognitive performance within the normal range (i.e., score higher than 1.5 SDs below the age- and education-adjusted norms in any cognitive test). In addition, they had no evidence of hippocampal atrophy on MRI (i.e., normal medial temporal lobe atrophy score) [46] and did not have a family history of neurodegenerative diseases in firstdegree relatives. These stringent criteria were applied to minimize the possibility of including participants with preclinical and early clinical neurodegenerative diseases.

Participants with depressive symptoms ( $\geq 6$ points on the 15 -item Geriatric Depression Scale (GDS-15)), anxiety ( $\geq 10$ points on the Beck Anxiety Inventory (BAI)), low visual acuity $(<20 / 40$ (corrected) on visual acuity tests), moderate to severe white matter vascular lesions on MRI (Fazekas score $>2$ points), and other primary neurological (history of stroke, Parkinson's disease, epilepsy, brain tumor) or psychiatric disorders [44] and those who did not successfully complete the training in the Navigation Test Suite were not included in the study.

\subsection{The Navigation Test Suite}

We used the Navigation test suite, which is described in detail in [38]. The Navigation Test Suite consists of three navigation tasks: The route-repetition task, the route-retracing task, and the directional-approach task. The Navigation Test Suite uses a virtual environment that consists of streets and four-way intersections with residential houses along the streets. The houses bordering the streets are all identical, except the unique houses (i.e., distinct landmarks) that are located at each intersection (explained in more detail below, Figure 1A,B). Participants can always see only one intersection at any time, because the other more distant intersections are concealed in white fog.

\section{(I) Route-repetition task}

In the encoding phase, the participants were positioned in a street next to a black car. They were then passively transported along a route featuring five intersections with one right turn, three left turns, and one straight movement. The route then stopped at a red phone box. Each intersection featured four identical houses at the four corners. Different intersections featured different houses, so that each intersection had a unique appearance. Participants were instructed to remember the route (Figure 1A).

In the test phase, the participants were asked to reproduce the same route from the car to the phone box. Participants were passively transported towards each of the intersections where they were stopped $20 \mathrm{~m}$ before the center of the intersections and prompted to verbally indicate the direction in which the route continued. The examiner pressed a corresponding arrow key, and the participants were passively transported to the center of the intersection facing the street, which led to the following intersection. Thus, participants did not receive feedback. The task was composed of three experimental sessions to assess learning.

(II) Route-retracing task

The encoding phase was similar to the route-repetition task, but the route was different and featured different houses at intersections (Figure 1A). In the test phase, the participants had to navigate in the opposite direction compared to the encoding phase, i.e., from the end point of the route (the telephone box) back to the start (the black car).

The route-repetition and route-retracing tasks consisted of 3 identical consecutive sessions and each session contained a route with 5 intersections. After completing each task, participants were asked to describe the strategy they used to solve the task. The reported 
strategies were then classified into three groups: (1) Sequence-of-directions, remembering a sequence of movement directions (e.g., left, right, straight, left, right) regardless of landmarks, (2) stimulus-response, creating associations between the places or landmarks with the movement direction (e.g., turn left at the intersection with blue houses), and (3) non-specific, such that participants reported inability to devise any strategy, or they used objects that could not be used as landmarks (e.g., observing clouds).

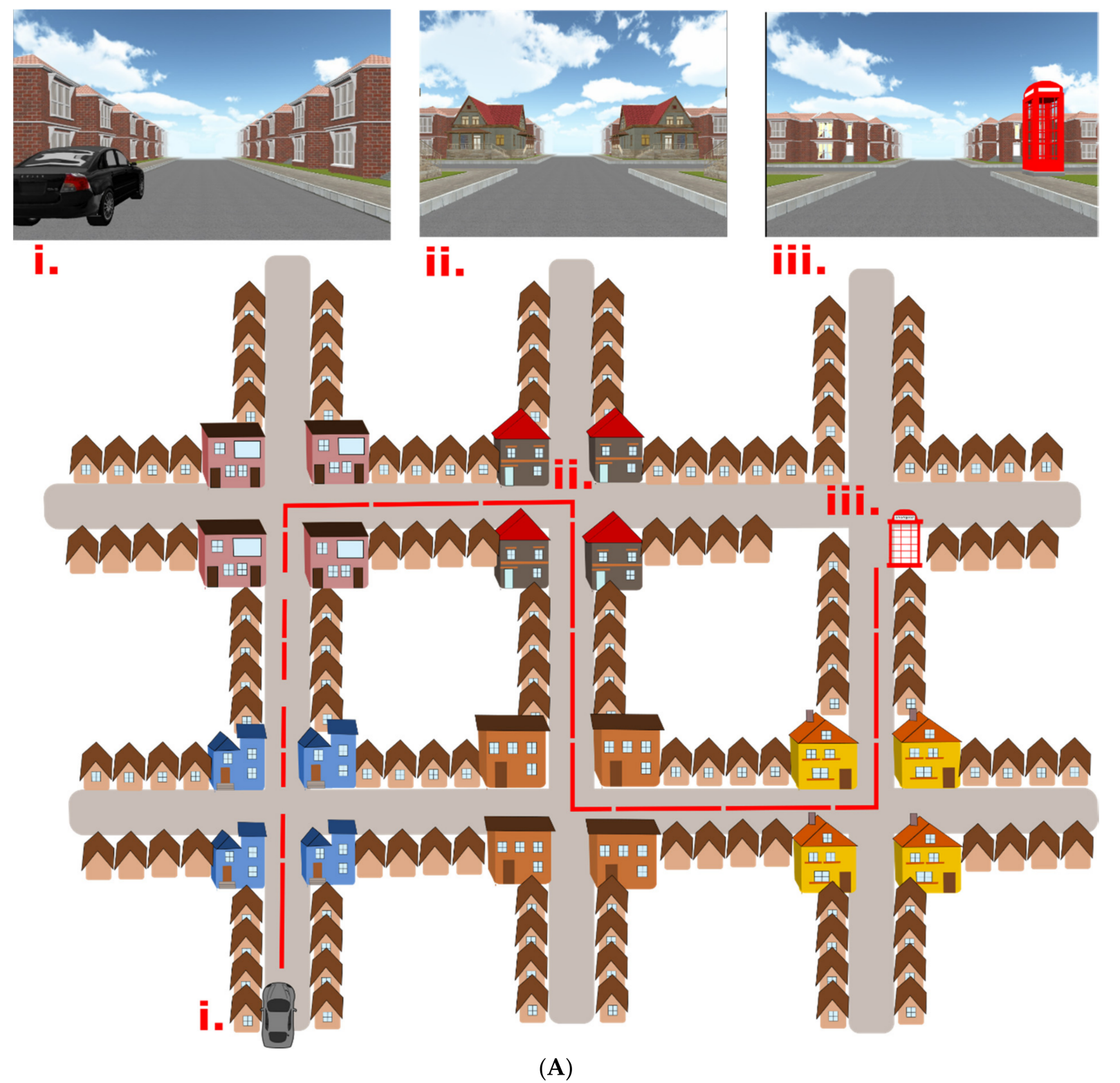

Figure 1. Cont. 


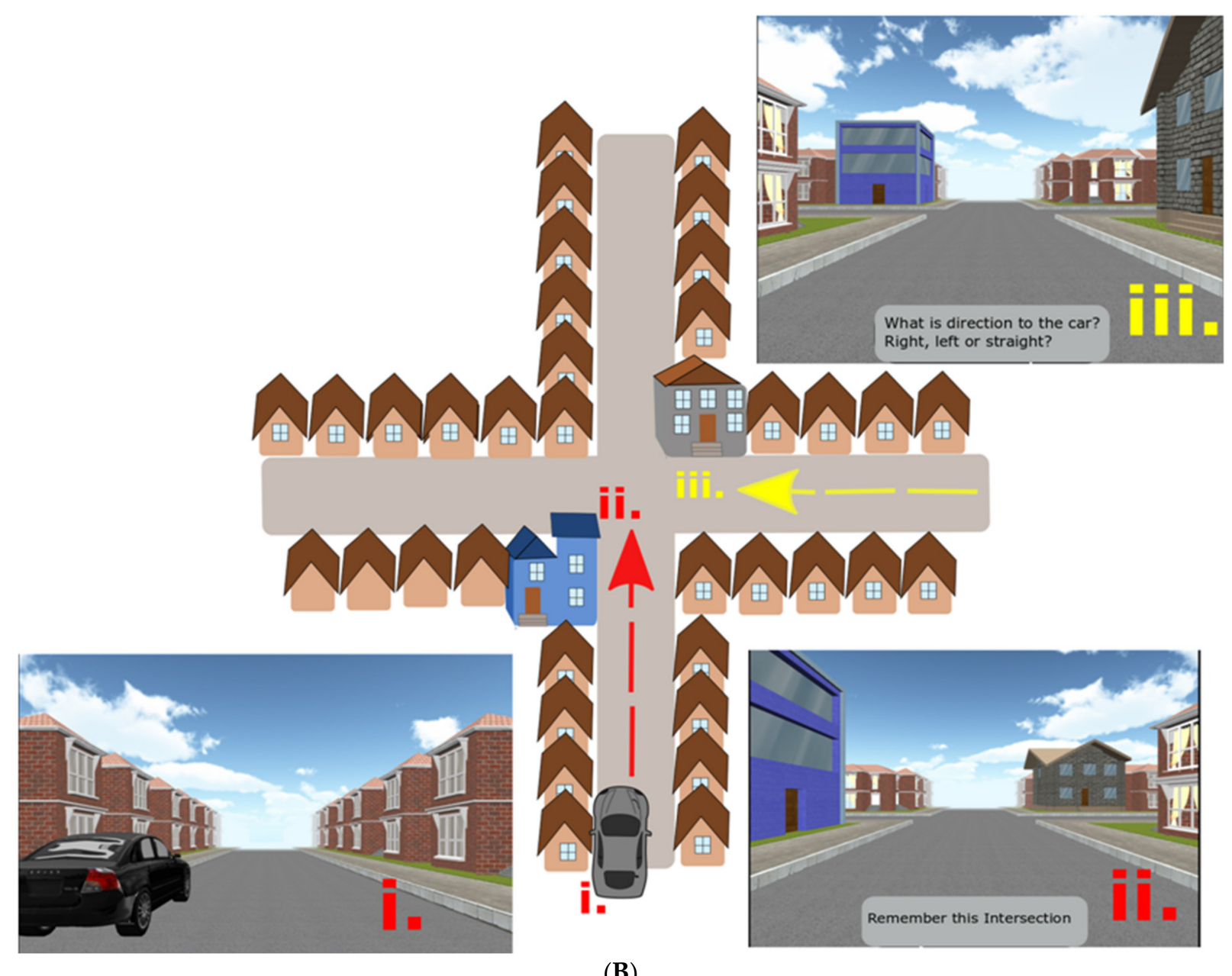

Figure 1. (A) The Navigation Test Suite with schematic aerial view and corresponding screenshots from the route-repetition and the route-retracing tasks. Three points on the map are labeled: (i.) The start location next to the car. (ii.) One of the intersections along the route with gray houses at the corners of the intersection. (iii.) The end of the route where the telephone box is present. In the route-repetition task, the participants were passively transported through the city from the car to the telephone box during the encoding phase and in the test phase the participants had to reproduce the same route. The route-retracing task was identical to the route-repetition task with the exception that participants in the test phase had to find their way back from the telephone box to the car. The order of intersections and houses at each intersection had a different design in each of these two tasks. (B) The Navigation Test Suite with schematic aerial view and corresponding screenshots from the directional-approach task: (i.) Participants started the task next to the car. (ii.) The encoding phase, where participants were passively transported towards one of the intersections featuring two unique houses. Participants had to remember where the car was parked. (iii.) The test phase, where participants approached the intersection from a different direction (here from east) and had to indicate direction to the car.

\section{(III) Directional-approach task}

This task tested participants' ability to encode the configuration of houses (landmarks) at an intersection and assessed wayfinding and perspective-taking abilities [48,49]. This task consisted of 15 independent trials. Each trial began with an encoding phase, in which participants were positioned in a street next to a black car from where they were passively transported toward a single intersection, which featured two unique houses (i.e., landmarks) at diagonally opposite corners of the intersection. The movement stopped $20 \mathrm{~m}$ before the center of the intersections, so both unique houses were in view. Two other houses at the corners of the intersection were identical with the other houses along the street. The participants' task was to memorize in which street the car was parked. Each 
of the 15 trials featured a different combination of unique houses at the corners of the intersections.

In the test phase, participants were passively transported toward the same intersection, but from one of the other streets. They were then asked to indicate the direction in which the car was parked (i.e., to indicate the street from which they originally approached the intersection). The movement stopped again $20 \mathrm{~m}$ before the center of the intersection, such that the unique houses could be seen.

The car was always parked in the street to the south of the intersection (Figure 1B). In the test phase, participants approached the intersection from the west, east, or north street. The participants were not aware of these cardinal directions in the experiment, but the information about these cardinal directions was used in the analysis. Participants were required to perform perspective shifts to align the view during the test phase with that during the perspective in the encoding phase. The perspective shift was $90^{\circ}$ when approaching the intersection from the west or east and $180^{\circ}$ when approaching from the north. As opposed to the previous route-repetition and route-retracing tasks, the directionalapproach task did not require participants to learn a route with multiple decision points.

Participants were asked about the navigation strategy after the Directional-approach task. Reported strategies were classified into three groups: (1) Unique houses, remembering the position of one or two unique houses at the intersections, (2) more houses, remembering three, all four, or non-unique houses, and (3) non-specific, (e.g., guessing, remembering grass, which was same at all intersections).

In all three tasks, participants indicated their responses verbally and the experimenter pressed the corresponding arrow key on the keyboard. A correct response at each of the intersections was counted as one point. Regarding the strategy analysis, participants were asked "How did you find the way?" at the end of each task. This was a very open question allowing the participant to describe their strategy in their own words. In the case when the response was not entirely clear, the participants were given another question for clarification "Could you please describe this in greater detail?". The participants were never asked suggestive questions. The examiner wrote down the participants' responses. Categories of navigation strategies were created retrospectively (when all responses were available) based on the given responses. Categorization was performed by two experienced examiners blinded to all other information about participants and their performance, each of the examiners classified the responses independently, and questionable responses were discussed with the supervisor.

Prior to the testing, all participants completed familiarization training consisting of shorter versions of all three tasks (a three-intersection path for the route-repetition and route-retracing tasks and two separate intersections for the directional-approach task) [38]. All CN participants and participants with aMCI and 51 out of 66 participants with mild AD dementia completed the training and all three tasks of the Navigation Test Suite. The participants who did not understand the training were not included in the study.

\subsection{Cognitive Assessment}

The cognitive assessment included the following tests: (1) Verbal memory measured with the Rey Auditory Verbal Learning Test-trials 1-5 and 30-min Delayed Recall trial and the Logical Memory I-Immediate and 20-min Delayed Recall conditions; (2) non-verbal memory measured with the Rey-Osterrieth Complex Figure Test-Recall condition after 3 min; (3) visuospatial function measured with the Rey-Osterrieth Complex Figure TestCopy condition and the Clock Drawing Test; (4) executive function measured with the Trail Making Test B, the Prague Stroop Test-colors, and the Controlled Oral Word Association Test (Czech version with letters N, K, and P); (5) attention and working memory measured with the Forward and Backward Digit Spans and the Trail Making Test A; and (6) language measured with the Boston Naming Test (30-item version) and the Category Fluency test (Animals). The Mini-Mental State Examination (MMSE) was administered to measure global cognitive function. The GDS-15 and BAI were used to assess depressive symptoms 
and anxiety among participants. Group-wise neuropsychological characteristics are listed in Table 1.

\subsection{Data Analysis}

For continuous demographic and cognitive variables, a one-way analysis of variance (ANOVA) with post hoc Sidak's test was used. For changes in proportion (gender and selected strategies), a $\chi^{2}$ test was used. Pearson's correlation with Holm-Bonferroni correction for multiple comparisons was used to assess associations between navigational tests and cognitive performance in each group. A two-way ANOVA and general linear models using the same data but focusing on different aspects of spatial navigation were performed for each task of the Navigation Test Suite and are described in detail below.

In the route-repetition and route-retracing tasks, a repeated-measures analysis of covariance (RM-ANCOVA) with the session (1st, 2nd, and 3rd) as the within-subjects factor and the group (CN, aMCI, and mild AD dementia) as the between-subjects factor was used to assess spatial navigation performance. Performance was measured as the percentage of trials in which participants provided a correct response. The analyses were controlled for age (mean-centered), years of education (mean-centered) and gender. Where applicable, the Greenhouse-Geisser and Huynd-Feldt corrections were used to correct for the violation of sphericity when epsilon was $\leq 0.75$ and $>0.75$, respectively. The planned polynomial contrasts were used to assess the effect of the session. The post hoc Sidak's test was used to assess differences between the individual groups and sessions. A onesample t-test was used to assess differences from chance performance (i.e., 33.33\%) for each group in each task and within each session. A two-way ANOVA with post hoc Sidak's test was used to evaluate the effect of group and reported strategy on spatial navigation performance. An RM-ANOVA with the order of intersections (1st, 2nd, 3rd, 4th, and 5th) as the within-subjects factor and group as the between-subjects factor was used to assess the serial position performance. Where applicable, the Greenhouse-Geisser and Huynd-Feldt corrections were used to correct for the violation of sphericity. The planned polynomial contrasts were used to assess the effect of the order of intersections and the post hoc Sidak's test was used to assess differences between the individual groups and orders of intersections.

In the directional-approach task, an RM-ANCOVA with the approach direction (west, north, and east) as the within-subjects factor and group (CN, aMCI, and mild AD dementia) as the between-subjects factor was used to assess spatial navigation performance. Again, the analysis was controlled for age (mean-centered), education (mean-centered), and gender, the Greenhouse-Geisser and Huynd-Feldt corrections were used to correct for the violation of sphericity (where applicable), the planned polynomial contrasts were used to assess the effect of approach direction, and the post hoc Sidak's test was used to assess differences between the individual groups and specific approach directions. A one-sample t-test was used to assess differences from chance performance (i.e., 33.33\%) for each group in the task and within each approach direction. A two-way ANOVA with post hoc Sidak's test was used to evaluate the effect of group and reported strategy on spatial navigation performance.

\section{Results}

\subsection{Demographic Characteristics}

The demographic characteristics are presented in detail in Table 1. The CN group was younger than the aMCI and mild AD dementia groups (both $p \leq 0.020$ ) and the aMCI group was younger than the mild AD dementia group $(p=0.001)$. The $\mathrm{CN}$ group was more educated that the aMCI and mild AD dementia groups (both $p \leq 0.028$ ). There were more women in the $\mathrm{CN}$ and mild AD dementia groups than in the aMCI group $(71.8 \%$ and $63.6 \%$ vs. $44.0 \%)\left[\chi^{2}(2)=43.21, p=0.002\right]$. As expected, the $\mathrm{CN}$ group had higher MMSE scores (both $p<0.001$ ) and showed better cognitive performance (both $p \leq 0.025$ ) than the 
$\mathrm{aMCI}$ and mild AD dementia groups. There were no differences in depressive and anxiety symptoms between the groups (all $p \geq 0.133$ ).

\subsection{Route-Repetition Task}

The RM-ANCOVA assessing spatial navigation performance and learning across the sessions revealed significant main effects of group $\left[F(2,193)=24.12, p<0.001, \eta_{\mathrm{p}}{ }^{2}=0.20\right]$ and session $\left[F(2,386)=41.69, p<0.001, \eta_{\mathrm{p}}{ }^{2}=0.18\right]$ (Figure 2). Specifically, the CN group performed better than the aMCI and mild AD dementia groups (both $p<0.001$ ) and the $\mathrm{aMCI}$ group performed better than the mild AD dementia group $(p=0.001)$. Performance in the second experimental session was better than that in the first experimental session $(p<0.001)$ and performance in the third experimental session was better than that in the first and second experimental sessions (both $p<0.001$ ). The interaction between group and experimental session was not significant $\left[F(4,386)=1.29, p=0.273, \eta_{\mathrm{p}}{ }^{2}=0.01\right]$. All groups performed above chance level in the task overall and within each session ( $\mathrm{CN}$ group: [all $t(77) \geq 12.76, p<0.001$ ]; aMCI group: [all $t(74) \geq 7.77, p<0.001$ ]; mild AD dementia group: [all $t(50) \geq 4.52, p<0.001])$. The effect of age $\left[F(1,193)=4.77, p=0.030, \eta_{\mathrm{p}}{ }^{2}=0.24\right]$ and education $\left[F(1,193)=4.50, p=0.035, \eta_{p}^{2}=0.23\right]$ was significant. The effect of gender $[F(1$, $\left.193)=0.73, p=0.395, \eta_{p}^{2}=0.00\right]$ and the gender-by-group interaction $[F(2,193)=0.80$, $\left.p=0.451, \eta_{\mathrm{p}}{ }^{2}=0.01\right]$ were not significant.

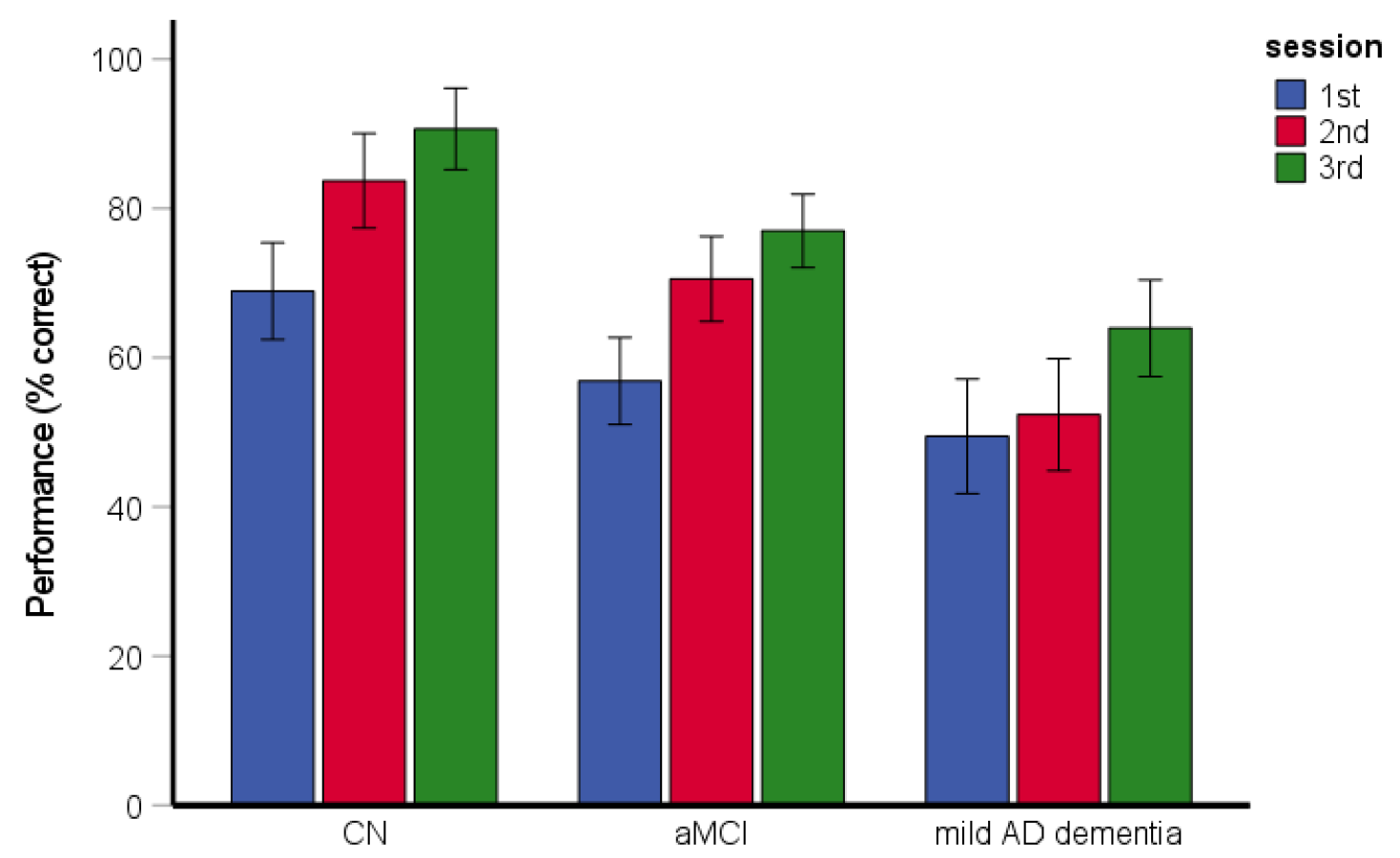

Figure 2. Route-repetition task—spatial navigation performance and learning across the three experimental sessions. CN, cognitively normal; aMCI, amnestic mild cognitive impairment; mild AD dementia, mild Alzheimer's disease dementia.

The $\chi^{2}$ test revealed the differences between the groups in reported strategies $\left[\chi^{2}(4)=43.21, p<0.001\right.$, Cramer's V $\left.=0.33\right]$. Specifically, the CN participants and participants with aMCI reported the use of the non-specific strategy less frequently than the stimulus-response and the sequence-of-directions strategies (75.3\% sequence-of-directions, $23.4 \%$ stimulus-response, $1.3 \%$ non-specific and $80.3 \%$ sequence-of-directions, $14.1 \%$ stimulus-response, $5.6 \%$ non-specific, respectively), while the participants with mild $\mathrm{AD}$ dementia reported the use of the non-specific and sequence-of-directions strategies with similar frequency ( $44.9 \%$ sequence-of-directions, $18.4 \%$ stimulus-response, $36.7 \%$ nonspecific). 
The two-way ANOVA assessing the effect of reported strategy on spatial navigation performance revealed significant main effects of group $\left[F(2,188)=5.50, p=0.005, \eta_{\mathrm{p}}{ }^{2}=0.06\right]$ and reported strategy $\left[F(2,188)=6.68, p=0.002, \eta_{\mathrm{p}}{ }^{2}=0.07\right]$ (Figure 3$)$. Specifically, the $\mathrm{CN}$ group performed better than the aMCI $(p=0.035)$ and mild AD dementia $(p=0.003)$ groups. The aMCI group did not differ from the mild AD dementia group $(p=0.735)$. The participants reporting the use of the non-specific strategy had worse performance than those reporting the use of the sequence-of-directions $(p=0.001)$ and the stimulus-response $(p=0.006)$ strategies. The performance did not differ between participants reporting the use of the sequence-of-directions and the stimulus-response strategies $(p=0.881)$. The interaction between group and reported strategy was significant $[F(4,188)=2.52, p=0.043$, $\left.\eta_{\mathrm{p}}{ }^{2}=0.05\right]$. Specifically, the participants with aMCI reporting the use of the non-specific strategy had worse performance than those reporting the use of the sequence-of-directions $(p<0.001)$ and stimulus-response $(p=0.004)$ strategies but the participants with mild AD dementia did not differ in performance with respect to the reported strategy (all $p \geq 0.201$ ). As there was only one $\mathrm{CN}$ participant reporting the use of the non-specific strategy, a post-hoc one-sample t-test was used. The test revealed that the $\mathrm{CN}$ participant reporting the use of the non-specific strategy had worse performance than those reporting the use of the sequence-of-directions $[t(57)=14.04, p<0.001]$ and stimulus-response $[t(17)=9.06$, $p<0.001$ ] strategies.

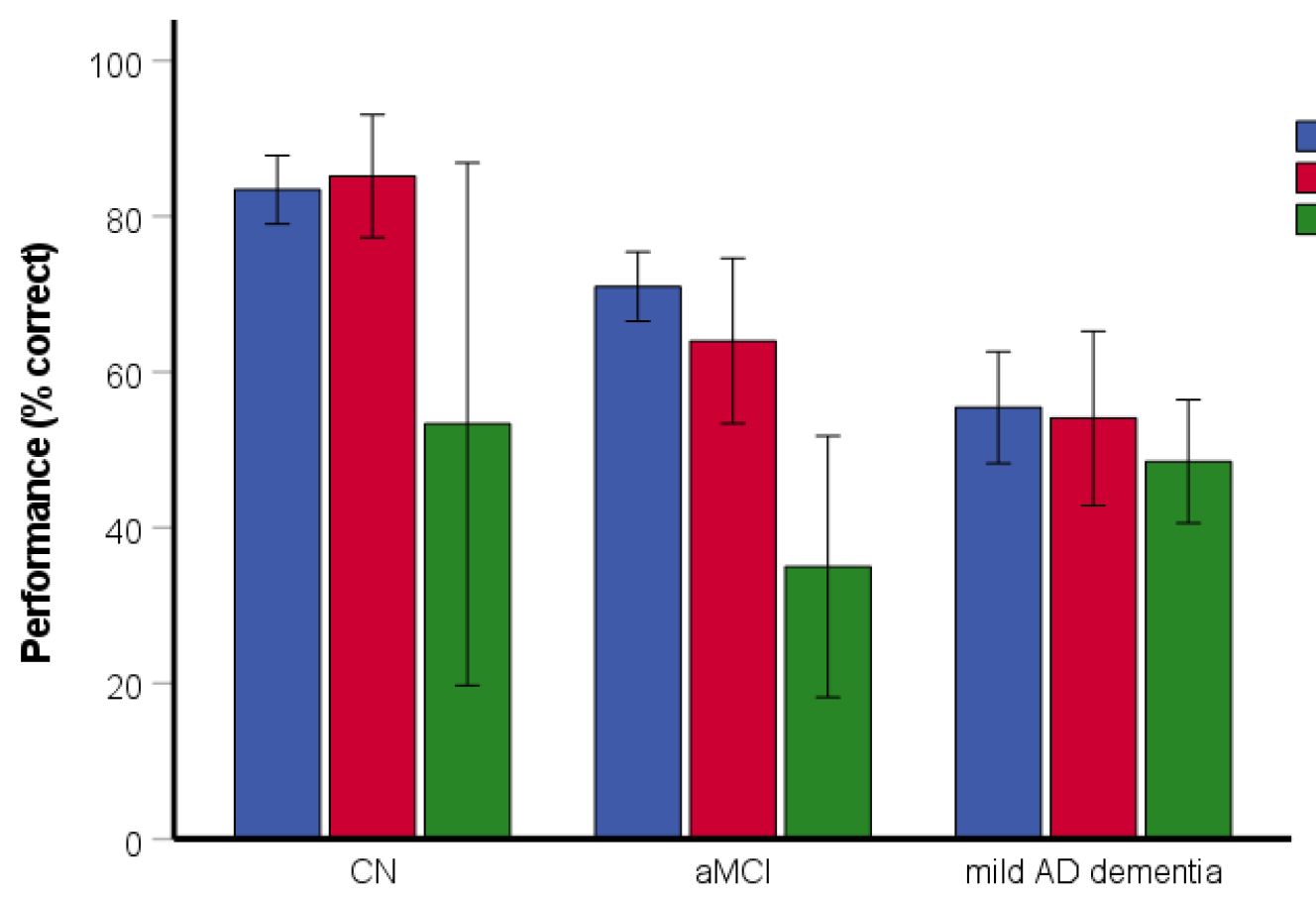

reported navigation strategy

Sequence-of-directions Stimulus-response Non-specific

Figure 3. Route-repetition task-the effect of reported strategy on spatial navigation performance. $\mathrm{CN}$, cognitively normal; aMCI, amnestic mild cognitive impairment; mild AD dementia, mild Alzheimer's disease dementia.

The RM-ANOVA assessing the serial position performance revealed significant main effects of group $\left[F(2,199)=47.06, p<0.001, \eta_{\mathrm{p}}{ }^{2}=0.32\right]$ and the order of intersections $[F(3.68$, $\left.731.26)=50.16, p<0.001, \eta_{p}^{2}=0.20\right]$ (Figure 4). Specifically, the $\mathrm{CN}$ group performed better than the aMCI and mild AD dementia groups (both $p<0.001$ ) and the aMCI group performed better than the mild AD dementia group $(p<0.001)$. Performance for the first intersection was better than that for the third (i.e., middle) intersection $(p<0.001)$ and the fifth (i.e., last) intersection $(p<0.001)$. Performance for the fifth intersection did not differ from that for the third intersection $(p=0.202)$. The interaction between group and order of intersections was significant $\left[F(7.35,731.26)=2.20, p=0.030, \eta_{\mathrm{p}}{ }^{2}=0.02\right]$. Specifically, the aMCI group performed worse for the fifth intersection than for the third intersection $(p=0.030)$ but performance for the fifth intersection did not differ from that for the third 
intersection in the CN ( $p=0.630)$ and mild AD dementia $(p=0.191)$ groups. In all groups, the performance for the first intersection was better than for the third (all $p<0.001)$ and fifth (all $p<0.001)$ intersections.

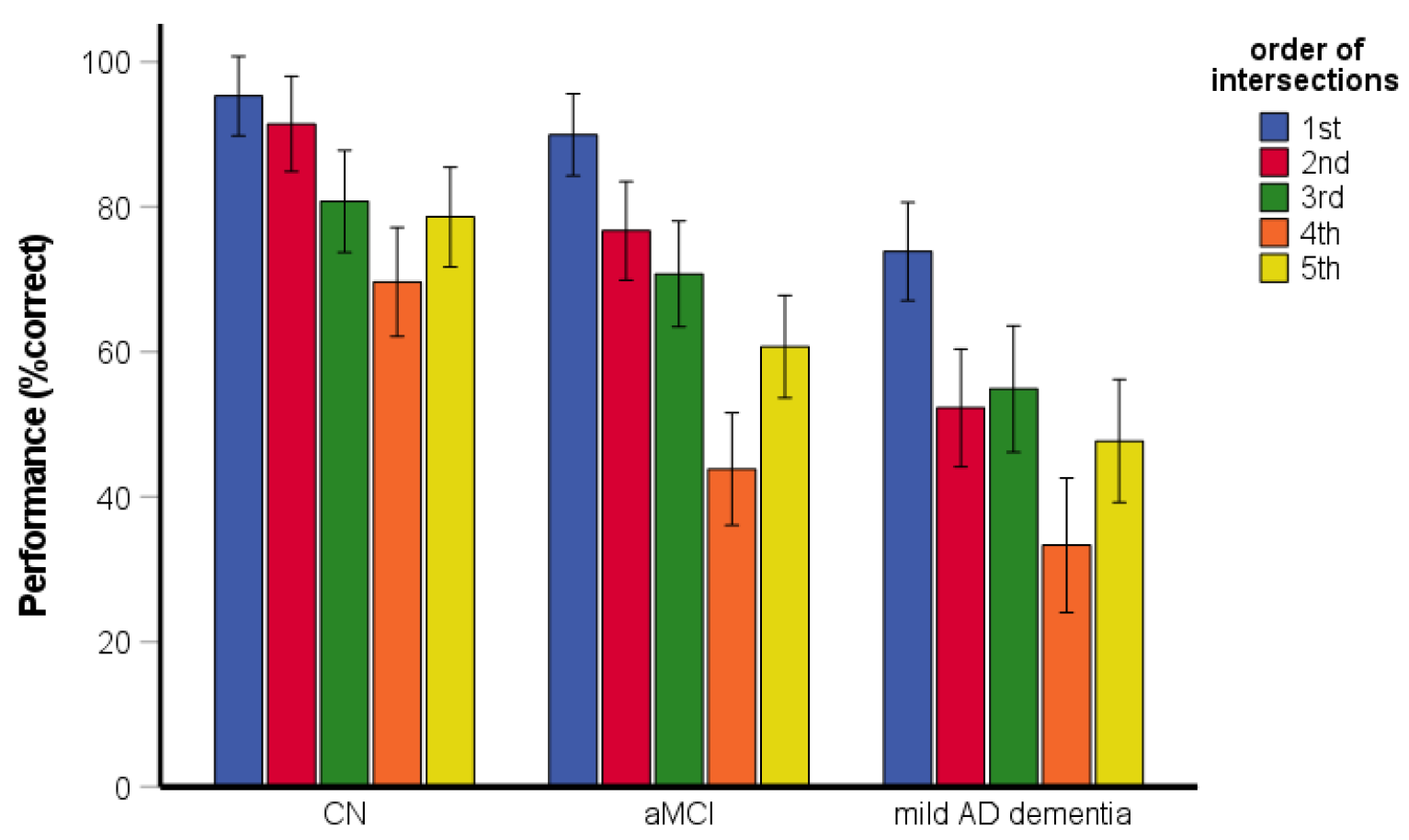

Figure 4. Route-repetition task-the serial position effect. $\mathrm{CN}$, cognitively normal; aMCI, amnestic mild cognitive impairment; mild AD dementia, mild Alzheimer's disease dementia.

\subsection{Route-Retracing Task}

The RM-ANCOVA assessing spatial navigation performance and learning across the sessions revealed significant main effects of group $\left[F(2,194)=14.99, p<0.001, \eta_{\mathrm{p}}{ }^{2}=0.13\right]$ and session $\left[F(2,388)=7.79, p<0.001, \eta_{\mathrm{p}}{ }^{2}=0.04\right]$ (Figure 5). Specifically, the CN group performed better than the $\mathrm{aMCI}(p=0.010)$ and mild AD dementia $(p<0.001)$ groups and the aMCI group performed better than the mild AD dementia group $(p=0.003)$. Performance in the third experimental session was better than that in the first $(p=0.001)$ and second $(p=0.044)$ experimental sessions. Performance in the second experimental session did not differ from that in the first experimental session $(p=0.290)$. The interaction between group and experimental session was significant $\left[F(4,388)=2.62, p=0.035, \eta_{\mathrm{p}}{ }^{2}=0.03\right]$. This interaction was driven by a significant increase in performance in the CN group across all sessions (session 1 vs. session 2: $p=0.046$; session 1 vs. session 3: $p<0.001$; session 2 vs. session 3: $p=0.009$ ), whereas performance in the aMCI group improved significantly only between the first and the third session (session 1 vs. session 3: $p=0.002$; session 1 vs. session 2: $p=0.137$; session 2 vs. session 3: $p=0.061$ ), and performance in the mild AD dementia group did not significantly improve across the sessions (all $p \geq 0.592$ ). The CN and aMCI groups performed above chance level (CN group: [all $t(77) \geq 6.92, p<0.001$ ]; aMCI group: [all $t(73) \geq 3.88, p<0.001]$ ), whereas performance (overall and within each session) in the mild AD dementia group did not differ from chance level [all $t(50) \leq 1.39, p \geq 0.170$ ]. The effect of age $\left[F(1,194)=4.47, p=0.036, \eta_{p}{ }^{2}=0.23\right]$ and education $[F(1,194)=3.95, p=0.048$, $\left.\eta_{\mathrm{p}}{ }^{2}=0.02\right]$ was significant. The effect of gender $\left[F(1,194)=0.97, p=0.326, \eta_{\mathrm{p}}{ }^{2}=0.01\right]$ and the gender-by-group interaction $\left[F(2,194)=0.37, p=0.692, \eta_{\mathrm{p}}{ }^{2}=0.00\right]$ were not significant. 


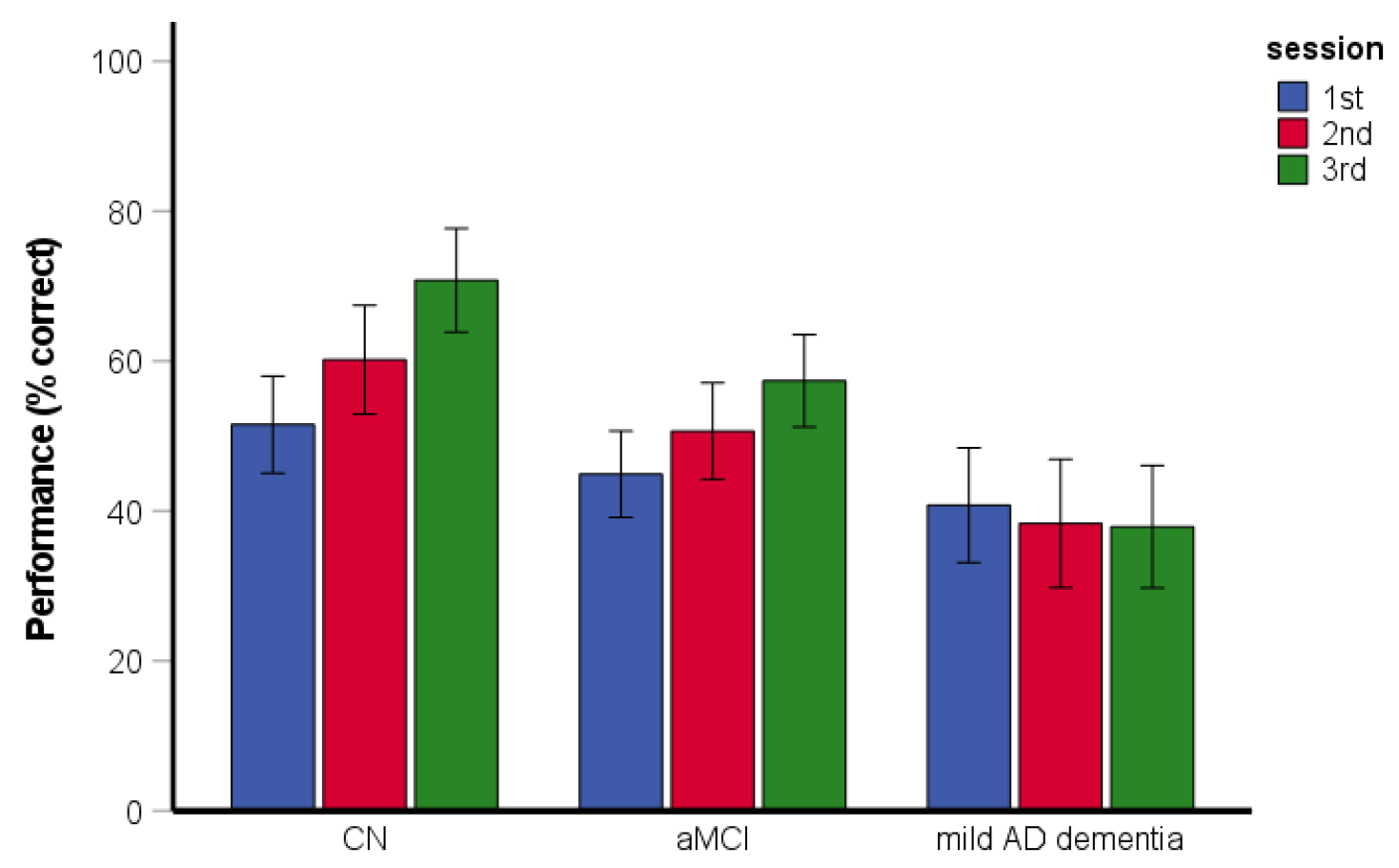

Figure 5. Route-retracing task-spatial navigation performance and learning across the sessions. CN, cognitively normal; aMCI, amnestic mild cognitive impairment; mild AD dementia, mild Alzheimer's disease dementia.

The $\chi^{2}$ test revealed differences between the groups in the reported strategies $\left[\chi^{2}(4)=27.70, p<0.001\right.$, Cramer's V $\left.=0.27\right]$. Specifically, the CN participants reported the use of the non-specific strategy less frequently than the stimulus-response and the sequence-of-directions strategies (70.1\% sequence-of-directions, $23.4 \%$ stimulus-response, $6.5 \%$ non-specific) and the participants with aMCI reported the use of the non-specific strategy less frequently than the sequence-of directions strategy (68.6\% sequence-of-directions, $17.1 \%$ stimulus-response, $14.3 \%$ non-specific), while the participants with mild AD dementia reported the use of the non-specific and the sequence-of-directions strategies with similar frequency (36.7\% sequence-of-directions, $22.4 \%$ stimulus-response, $40.8 \%$ non-specific).

The two-way ANOVA assessing the effect of the reported strategy on spatial navigation performance revealed significant main effects of group $[F(2,187)=15.39, p<0.001$, $\left.\eta_{\mathrm{p}}{ }^{2}=0.14\right]$ and reported strategy $\left[F(2,187)=3.99, p=0.020, \eta_{\mathrm{p}}{ }^{2}=0.04\right]$ (Figure 6). Specifically, the $\mathrm{CN}$ group performed better than the aMCI $(p=0.031)$ and mild AD dementia $(p<0.001)$ groups and the aMCI group performed better than the mild AD dementia group $(p=0.006)$. The participants reporting the use of the non-specific strategy showed worse performance than those reporting the use of the sequence-of-directions strategy $(p=0.018)$. Differences in performance between participants reporting the use of the non-specific strategy and the stimulus-response strategy did not reach statistical significance $(p=0.065)$. The performance did not differ between participants reporting the use of the sequenceof-directions and the stimulus-response strategies $(p=0.998)$. The interaction between group and reported strategy did not reach statistical significance $[F(4,187)=2.23, p=0.068$, $\left.\eta_{\mathrm{p}}{ }^{2}=0.05\right]$. 


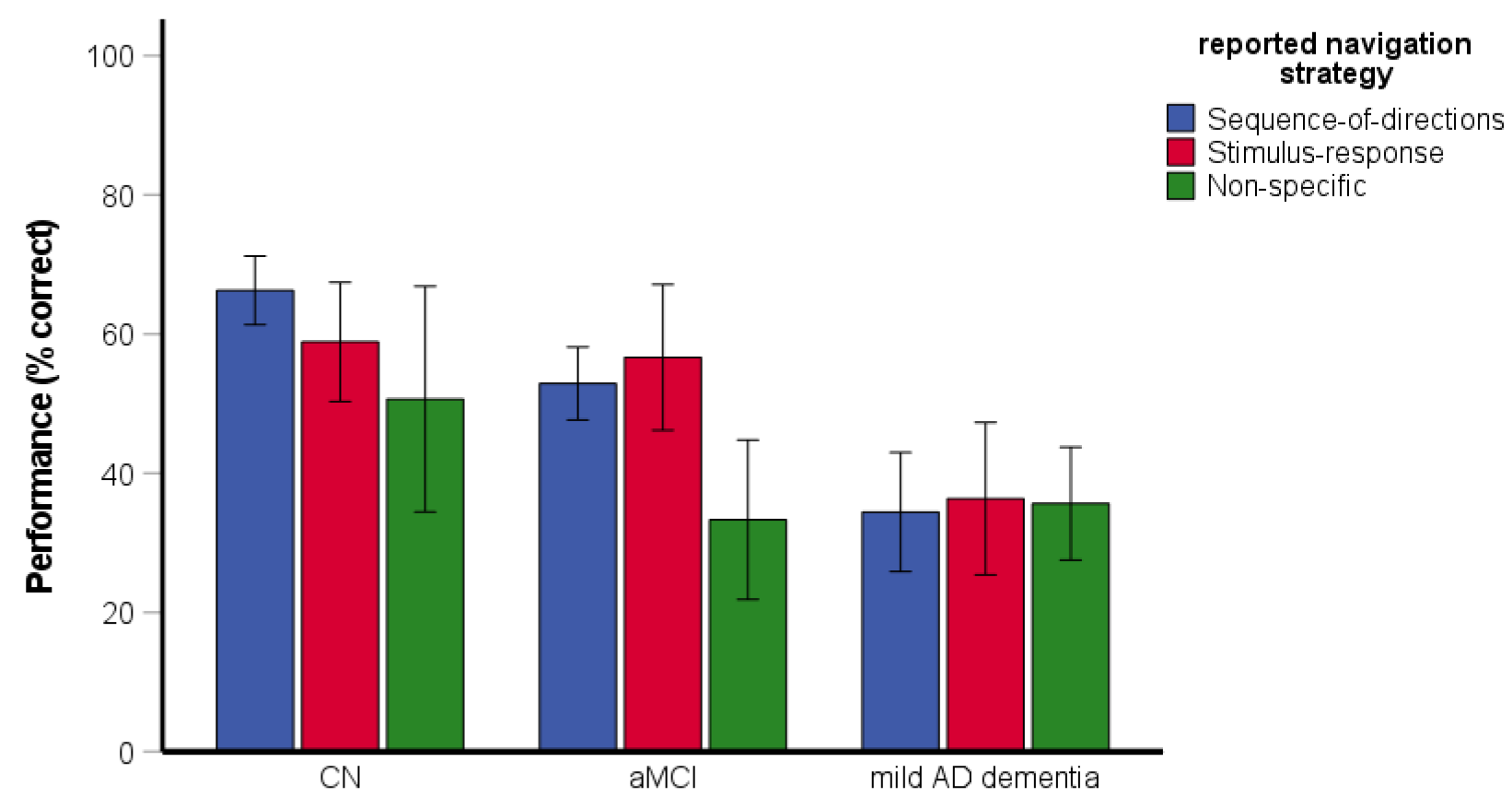

Figure 6. Route-retracing task-the effect of reported strategy on spatial navigation performance. CN, cognitively normal; aMCI, amnestic mild cognitive impairment; mild AD dementia, mild Alzheimer's disease dementia.

The RM-ANOVA assessing the serial position performance revealed significant main effects of group $\left[F(2,200)=32.54, p<0.001, \eta_{\mathrm{p}}{ }^{2}=0.25\right]$ and the order of intersections $[F(3.82$, 763.03 ) $=40.26, p<0.001, \eta_{\mathrm{p}}{ }^{2}=0.17$ ] (Figure 7). Specifically, the $\mathrm{CN}$ group performed better than the aMCI and mild AD dementia groups $(p<0.001)$ and the aMCI group performed better than the mild AD dementia group $(p<0.001)$. Performance for the fifth intersection based on the order of intersections in the encoding phase (i.e., the first intersection in the test phase) was better than that for the third (i.e., middle) intersection $(p<0.001)$ and the first intersection (i.e., the fifth intersection in the test phase) $(p<0.001)$. Performance for the first intersection did not differ from that for the third intersection $(p=1.000)$. The interaction between group and order of intersections was significant $[F(7.63,763.03)=2.40$, $\left.p=0.016, \eta_{\mathrm{p}}^{2}=0.02\right]$. Specifically, performance for the first intersection was better than for the third intersection in the $\mathrm{CN}$ group $(p=0.014)$ but performance for the first intersection did not differ from that for the third intersection in the aMCI $(p=0.175)$ and mild AD dementia $(p=0.744)$ groups. Performance for the fifth intersection was better than for the third (all $p \leq 0.007$ ) and first (all $p \leq 0.004$ ) intersections in all groups.

\subsection{Directional-Approach Task}

The RM-ANCOVA assessing spatial navigation performance and the effect of approach direction on navigational performance revealed significant main effects of group $\left[F(2,193)=18.21, p<0.001, \eta_{\mathrm{p}}{ }^{2}=0.16\right]$ and approach direction $[F(1.82,350.92)=81.11$, $p<0.001, \eta_{\mathrm{p}}{ }^{2}=0.30$ ] (Figure 8). Specifically, the CN group performed better than the aMCI and the mild AD dementia groups (both $p<0.001$ ) and the aMCI group did not differ from the mild AD dementia group $(p=0.222)$. Performance was worse for an approach from the north than for an approach from the east $(p<0.001)$ and the west $(p<0.001)$. Performance for the west and east approaches did not differ from each other $(p=0.875)$. The interaction between group and approach direction was not significant $[F(3.64,350.92)=0.43, p=0.772$, $\left.\eta_{\mathrm{p}}^{2}=0.00\right]$. For an approach from the north, the CN group performed above chance level $[t(77)=3.05, p=0.003]$, whereas performance in the aMCI group did not differ from chance level $[t(72)=-1.07, p=0.289]$ and the mild AD dementia group performed below chance level $[t(50)=-4.13, p<0.001]$. All groups performed above chance level in the task overall and for approaches from the east and the west (CN group: [all $t(77) \geq 13.55, p<0.001$ ]; 
aMCI group: [all $t(72) \geq 6.87, p<0.001$ ]; mild $\mathrm{AD}$ dementia group: [all $t(50) \geq 2.79$, $p \leq 0.008])$. The effect of age was significant $\left[F(1,193)=8.79, p=0.003, \eta_{p}^{2}=0.04\right]$, while the effect of education was not significant $\left[F(1,193)=1.02, p=0.313, \eta_{\mathrm{p}}{ }^{2}=0.01\right]$. The effect of gender was significant $\left[F(1,193)=8.55, p=0.004, \eta_{p}^{2}=0.04\right]$, where women generally had worse performance than men. The gender-by-group interaction $[F(2,193)=0.98$, $\left.p=0.378, \eta_{\mathrm{p}}^{2}=0.01\right]$ was not significant.

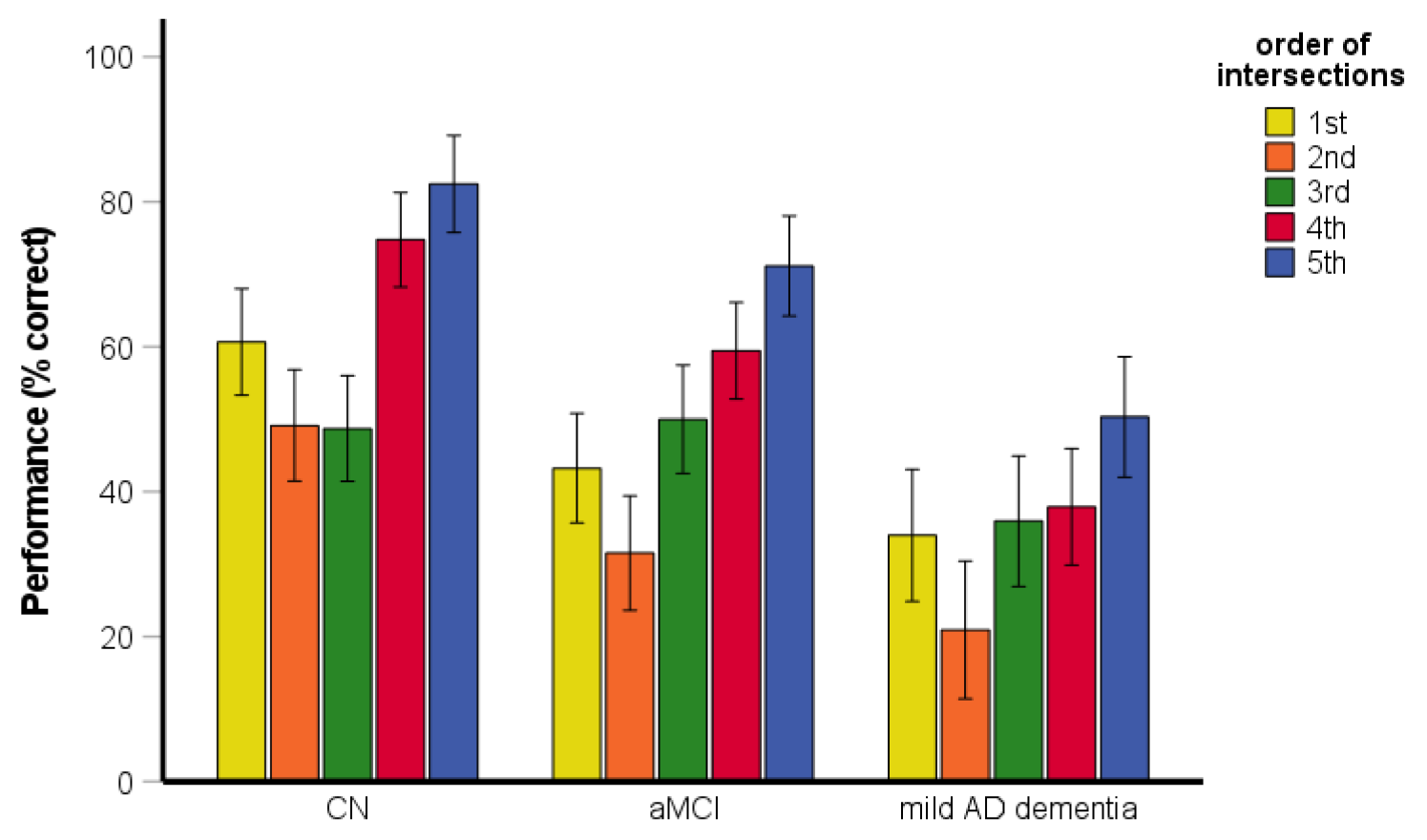

Figure 7. Route-retracing task-the serial position effect. Intersections in the route-retracing task were recalled in reverse order than in which they were presented during the encoding phase. Order of intersections refers to the order in the test phase (i.e., the 1st intersection that was first recalled in the test phase was the last encoded during the encoding phase). $\mathrm{CN}$, cognitively normal; aMCI, amnestic mild cognitive impairment; mild AD dementia, mild Alzheimer's disease dementia.

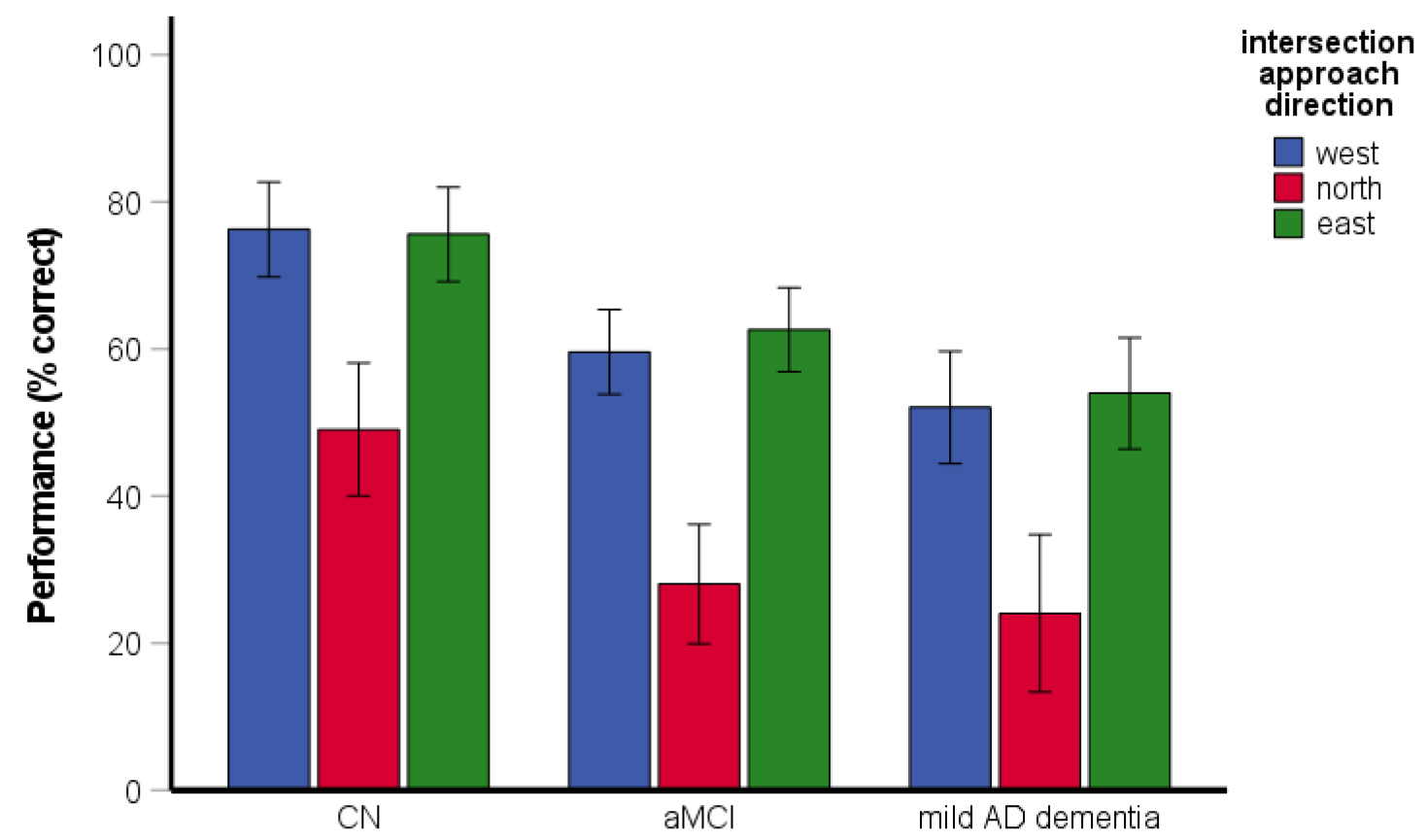

Figure 8. Directional-approach task—spatial navigation performance and the effect of approach direction. CN, cognitively normal; aMCI, amnestic mild cognitive impairment; mild AD dementia, mild Alzheimer's disease dementia. 
The $\chi^{2}$ test revealed differences between the groups in reported strategies $\left[\chi^{2}(4)=53.45, p<0.001\right.$, Cramer's V $\left.=0.37\right]$. Specifically, the CN participants reported the use of the unique-houses strategy more frequently than the more-houses and non-specific strategies (64.5\% unique houses, 30.3\% more houses, 5.3\% non-specific), while the participants with aMCI reported the use of the unique-houses and more-houses strategies with similar frequency (52.9\% unique houses, $44.3 \%$ more houses, $2.9 \%$ non-specific). The participants with mild AD dementia reported the use of the non-specific strategy more frequently than the unique-houses and the more-houses strategies (28.6\% unique houses, $26.5 \%$ more houses, $44.9 \%$ non-specific).

The two-way ANOVA assessing the effect of reported strategy on spatial navigation performance revealed significant main effects of group $\left[F(2,186)=8.60, p<0.001, \eta_{\mathrm{p}}{ }^{2}=0.09\right]$ and reported strategy $\left[F(2,186)=12.19, p<0.001, \eta_{p}{ }^{2}=0.12\right]$ (Figure 9). Specifically, the CN group performed better than the aMCI $(p=0.046)$ and the mild AD dementia $(p<0.001)$ groups and the aMCI group did not differ from the mild AD dementia group $(p=0.841)$. The participants reporting the use of the unique-houses strategy showed better performance than those reporting the use of the more-houses $(p<0.001)$ and non-specific $(p=0.002)$ strategies. Performance did not differ between the participants reporting the use of the more-houses and non-specific strategies $(p=0.534)$. The interaction between group and reported strategy did not reach statistical significance $\left[F(4,186)=2.40, p=0.052, \eta_{\mathrm{p}}{ }^{2}=0.05\right]$.

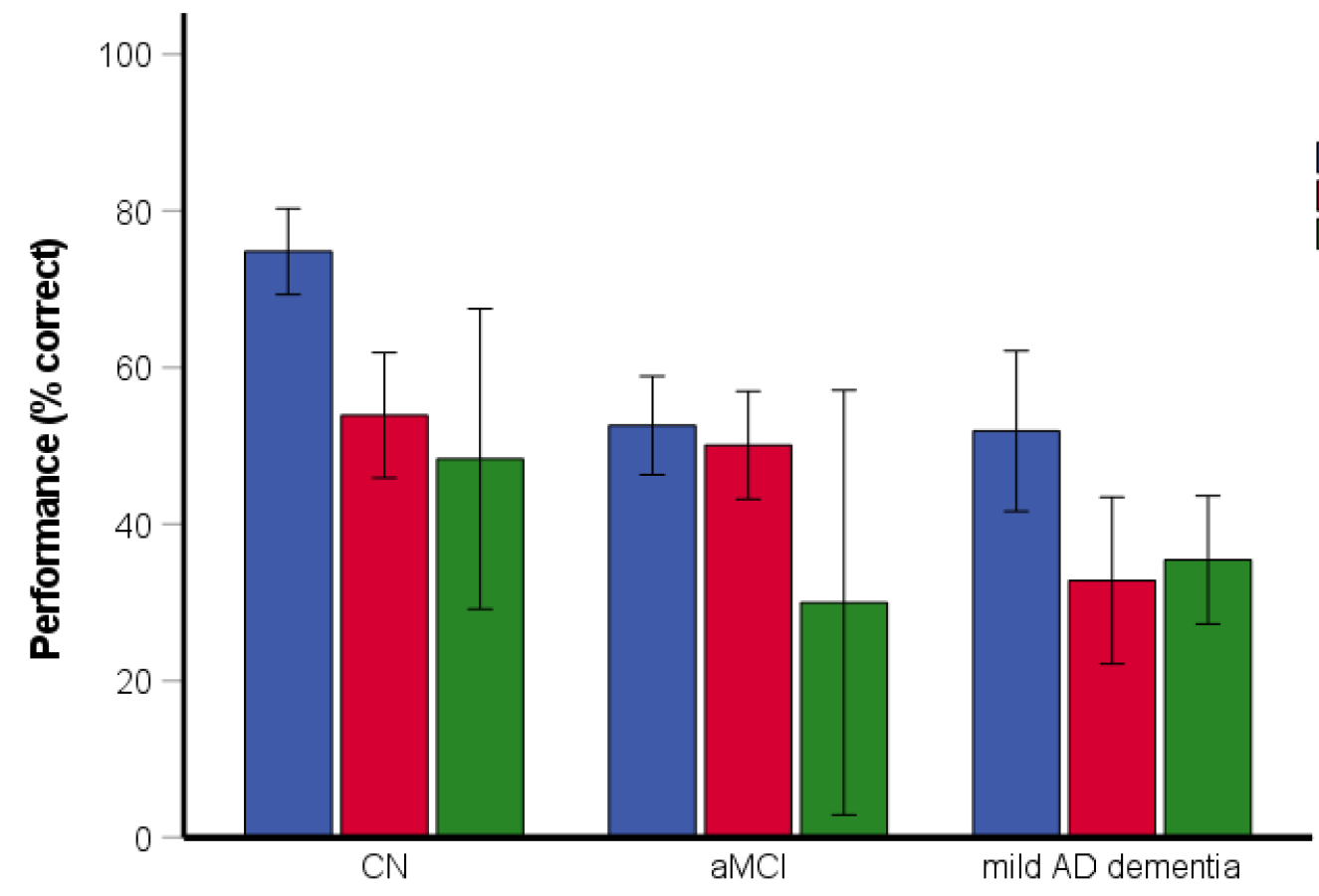

Figure 9. Directional-approach task—the effect of reported strategy on spatial navigation performance. CN, cognitively normal; aMCI, amnestic mild cognitive impairment; mild AD dementia, mild Alzheimer's disease dementia.

\subsection{Correlations between Navigational Tasks and Cognitive Performance}

Pearson's correlation with Holm-Bonferroni correction for multiple comparisons revealed a significant correlation between the route-repetition, route-retracing, and directionalapproach tasks in the aMCI group $(\mathrm{r}=0.39-0.49$, all $p \leq 0.001)$. The correlation was not significant or did not survive the adjustment for multiple comparisons in the CN group $(\mathrm{r}=0.20-0.26, p=0.075-0.022)$ and was not significant in the mild AD dementia group $(\mathrm{r}=-0.09--0.19$, all $p \geq 0.179)$. Because all three navigational tasks correlated with each other and to minimize a Type II error due to the correction for multiple comparisons, their scores were combined into a single total navigational score for correlational analysis with cognitive performance. In the analysis, the total navigational score correlated with verbal 
and non-verbal memory, executive and language functions in the $\mathrm{CN}$ group $(\mathrm{r}=0.30-0.35$, $p=0.002-0.007)$, verbal and non-verbal memory in the aMCI group $(\mathrm{r}=0.23, p=0.005$ and $\mathrm{r}=0.51, p<0.001$, respectively), and executive function in the mild AD dementia group $(\mathrm{r}=0.47, p=0.001)$.

\section{Discussion}

This study aimed to comprehensively explore spatial navigation in CN older adults and patients with aMCI and mild AD dementia. Specifically, we examined the differences in spatial navigation performance and navigation strategy selection, evaluated the effect of strategy selection on navigational performance, and analyzed learning across the experimental sessions, the effect of perspective shift on navigational performance, the serial position (i.e., primacy/recency) effect, and the associations between spatial navigation and cognitive performance. For this purpose, we used three navigational tasks in a realisticlooking virtual city addressing the ability to learn a route, the ability to retrace a recently travelled route, and the ability to learn and use a configuration of landmarks. Participants were interviewed about the use of navigation strategies to investigate visuospatial perception and its influence on spatial navigation performance.

In the route-repetition task, we observed route-learning deficits in aMCI patients that were even more pronounced in patients with mild AD dementia. This is in line with previous studies that reported route-learning impairment in MCI patients [24,50,51] and patients with AD [17,19,50-52] and a study showing more severe route-learning impairment in AD patients than those with MCI [50]. All groups showed learning across three experimental sessions. Learning in CN older adults in our study is in agreement with a previous study using the same experimental task that reported learning over the course of the experiment in older participants [38]. Learning in participants with a cognitive deficit that we have observed in the current study was not shown in previous studies using route-learning tasks in patients with aMCI [24] and AD [19]. It should be noted that these previous route-learning studies used longer routes with six and twelve intersections, respectively, compared to our route that consisted of five intersections. Our findings indicate that patients with aMCI and mild AD dementia are able to learn unfamiliar routes when the number of intersections does not exceed the spatial span of AD patients [53].

Participants most frequently reported that they remembered a sequence of turns regardless of landmarks (i.e., the sequence-of-directions strategy) and less frequently that they created associations between landmarks and movement directions (i.e., the stimulusresponse strategy). Only very few of the $\mathrm{CN}$ older adults and aMCI patients reported that they did not devise any specific strategy (i.e., non-specific strategy). In contrast, the sequence-of-directions strategy and the non-specific strategy were the most frequently reported strategies in patients with mild AD dementia. None of the participants reported creating a cognitive map to solve this route-learning task.

Route-learning performance was similar when using the sequence-of-direction strategy and the stimulus-response strategy. This is not surprising since both of these strategies are referred to as egocentric strategies because they rely on spatial information encoded in an egocentric reference frame [54]. The use of the non-specific strategy compared to adopting any of the egocentric strategies was associated with worse task performance, especially in CN older adults and aMCI patients. This finding is in line with a previous study that showed better route-learning performance in aMCI patients using a specific strategy compared to those using no specific strategy [24] and underlines the important role of the sequence-of-direction and stimulus-response strategies in route learning [1].

To the best of our knowledge, this is the first study analyzing serial position effects in a route-learning task in older adults with aMCI and mild AD dementia. We observed a primacy effect in all participant groups, where participants showed the best performance in the first intersection. Such a serial position effect has been reported in previous navigation studies $[55,56]$. A recent route-learning study showed the serial position effect for landmarks encountered during navigation with strong primacy and recency benefits in $\mathrm{CN}$ 
older adults [41]. In patients with MCI and AD dementia, list-learning tasks that are used to investigate serial position effects usually show a diminished primacy effect (for review see [57]), which was not observed in our navigational study. These discordant findings could be caused by different experimental protocols used in the current study and previous studies, which require one to recall directions at each intersection in the same order as during encoding in the route-learning task and allow one to recall words in any order in the list-learning tasks, respectively. Further studies are needed to investigate whether these methodological differences can account for differences in serial position effects in cognitively impaired older adults in word-list and route-learning studies.

In the route-retracing task, where participants navigated from the end of the route back to the start location, we observed navigation deficits in aMCI patients that were even more pronounced in patients with mild AD dementia. This finding is consistent with a previous study showing navigation deficits in patients with $\mathrm{MCI}$ and mild $\mathrm{AD}$, which were more frequent in the latter group, when navigating a route in the reverse direction in a real-space hospital lobby [50]. CN older adults gradually improved their performance across three experimental sessions, while patients with aMCI showed slower learning as they only improved between the first and last sessions. Patients with mild AD dementia did not show any learning and performed at chance level in all sessions. Successful navigation in this task requires creating a mental representation of the environment or the ability to imagine the environment from different viewpoints as egocentric strategies do not directly support route retracing [38]. About $94 \%$ of $\mathrm{CN}$ older adults, $86 \%$ of aMCI patients, and $59 \%$ of patients with mild AD dementia reported that they remembered a sequence of turns (i.e., the sequence-of-directions strategy) or created associations between landmarks and movement directions (i.e., the stimulus-response strategy) in the encoding phase and mirrored the direction of the turn required at each intersection when retracing a route, whereas none of the participants reported creating a cognitive map to solve the task. Worse performance in participants with cognitive deficits in this retracing task may thus be caused by the inaccurate alignment of the viewpoint in the test phase with the encoded viewpoint, i.e., by perspective shift deficits that have been reported in patients with aMCI and mild $\mathrm{AD}$, with more pronounced impairment in the latter group [28].

It is noteworthy that $41 \%$ of patients with mild AD dementia did not report any specific strategy (i.e., used the non-specific strategy). The use of this non-specific strategy was associated with worse task performance compared to adopting the sequence-of-directions strategy. Therefore, the inability to adopt an effective non-egocentric strategy to solve the task could contribute to severe route-retracing deficits and the absence of learning in patients with mild AD dementia, and is in line with previous findings of a strong preference for egocentric (i.e., extrahippocampal) strategies in AD patients [26].

We also observed serial position effects in this task. Specifically, we found a recency effect in all groups, where participants showed the best performance in the last encoded (i.e., first recalled) intersection. In addition, we observed a primacy effect in $\mathrm{CN}$ older adults with better performance in the first encoded (i.e., last recalled) intersection. To the best of our knowledge, the serial position effect has not been studied in route-retracing tasks. A recent study using a different paradigm reported primacy and recency effects for landmarks in a route-learning task in $\mathrm{CN}$ older adults [41]. Our findings of prominent recency and reduced primacy effects in cognitively impaired older adults are in line with studies showing reduced primacy effect in verbal memory tests in patients with MCI and $\mathrm{AD}$ dementia [58] and in non-verbal memory tests in the latter group [59].

In the directional-approach task, the participants were required to encode spatial relationships of landmarks (houses) at an intersection in relation to the direction from which the intersection was approached originally. Here we observed navigation deficits in patients with aMCI and mild AD dementia compared to $\mathrm{CN}$ older adults. This is in line with a previous study showing navigation deficits in patients with $\mathrm{MCI}$ and mild $\mathrm{AD}$ that were more pronounced when approaching an intersection from a different direction than during learning [50]. In all groups, performance decreased when the approach direction 
in the test phase was misaligned with the encoding phase by $180^{\circ}$ compared to $90^{\circ}$. This corroborates similar findings of a previous study with the same paradigm in $\mathrm{CN}$ older adults [38] and supports earlier notions that perspective-taking abilities are required to solve this task [48]. It should be noted that patients with aMCI and mild AD dementia performed at and below the chance level, respectively, when the direction in the test phase was misaligned by $180^{\circ}$. Our findings thus indicate that patients with aMCI and mild AD dementia show perspective-taking deficits similar to what was previously reported in a virtual arena task [28].

The $\mathrm{CN}$ normal older adults most frequently reported that they remembered the positions of unique houses at the intersection (i.e., the unique-houses strategy), while aMCI patients reported with a similar frequency that they used unique houses and nonunique houses or more than two houses (i.e., unique-houses and more-houses strategies). Almost half of the patients with mild AD dementia did not report any specific strategy (i.e., used the non-specific strategy). It is important to note that the use of the unique-houses strategy compared to the adoption of any other strategy was associated with better task performance. Using an effective strategy was associated with better performance even in the mild AD dementia group. These findings indicate that successful navigation in this task requires an engagement of allocentric processes including knowledge about the spatial relationships between landmarks (i.e., unique houses) and a place (i.e., street from which the intersection was approached) that were reported to be impaired in patients with aMCI and mild AD dementia [26].

Further, we assessed differences in spatial navigation performance in women and men showing that gender did not influence performance in route-repetition and route-retracing tasks. However, the effect of gender was found in the directional-approach task where women had worse performance than men across all diagnostic groups. This is consistent with our previous findings where the perspective-taking deficit was more pronounced in women than men [28].

Finally, we explored the associations of spatial navigation performance between the three navigation tasks and with cognitive functions. We found correlations between the tasks in aMCI patients but not in CN older adults and patients with mild AD dementia. This finding may reflect greater variability of performance in aMCI patients and supports the previously described heterogeneity of $\mathrm{MCI}$ patients who may greatly vary with respect to spatial navigation performance $[18,60]$ and the number, type, and severity of impairment of individual cognitive functions $[47,61]$. Spatial navigation performance correlated with memory, executive, and language functions in $\mathrm{CN}$ older adults, memory in aMCI patients, and executive function in patients with mild AD dementia. These findings indicate that specific cognitive resources including the use of effective memory strategies [62] and executive skills (e.g., planning and strategy selection) [63] may contribute to successful spatial navigation. They are also congruent with previous research associating more effective spatial navigation in various tasks with better memory, executive, and language functioning in $\mathrm{CN}$ older adults [63-67] and better memory and executive functioning in patients with $\mathrm{MCI}$ and $\mathrm{AD}$ dementia $[51,68,69]$.

The strengths of the current study are the fact that we explored three different spatial navigation tasks that rely on different cognitive processes in the same participants. Specifically, we recruited a sufficient number $(n=219)$ of clinically well-defined $\mathrm{CN}$ older adults and patients with cognitive impairment including patients with aMCI and mild AD dementia. We also used an established, realistic-looking, and ecologically valid method for spatial navigation testing, the Navigation Test Suite, along with detailed cognitive assessment. However, this study is not without its limitations. First, we have not examined structural and functional alterations in specific brain regions that support spatial navigation to reveal the underlying mechanisms of the observed behavioral findings, which should be a focus of future studies. Second, we have not analyzed specific biomarkers including cerebrospinal fluid analysis and amyloid or tau positron emission tomography imaging to confirm the clinical diagnosis of $\mathrm{AD}$ in patients with mild dementia and $\mathrm{aMCI}$. Third, 
in each task, we used separate ANCOVA analyses for each hypothesis, although using one ANCOVA would be a more appropriate statistical method. However, such analysis would make the results difficult to interpret and, importantly, it would significantly reduce the statistical power in our sample size. Fourth, the cross-sectional design did not allow for evaluating the changes in spatial navigation performance over time, but longitudinal follow-up is ongoing.

\section{Conclusions}

In conclusion, the current study demonstrated spatial navigation deficits in route learning, route retracing, and wayfinding/perspective-taking in patients with aMCI that are even more pronounced in patients with mild AD dementia. $\mathrm{CN}$ older adults were able to learn in both route-learning and route-retracing tasks, while patients with aMCI and mild $\mathrm{AD}$ dementia were able to gradually improve their performance only in a route-learning task when the number of intersections did not exceed the spatial span of AD patients. Patients with aMCI showed slower learning in a route-retracing task, while patients with mild AD dementia did not learn and performed at chance level throughout the experiment. Greater misalignment in a wayfinding/perspective-taking task was associated with worse performance in both typical and atypical aging. $\mathrm{CN}$ older adults and aMCI patients were able to adopt specific strategies in route-learning and route-retracing tasks, while patients with mild $\mathrm{AD}$ dementia more frequently used non-specific strategies. Patients with aMCI used specific landmarks for navigation in a wayfinding/perspective taking task less frequently than $\mathrm{CN}$ older adults. This was even more pronounced in patients with mild AD dementia. Adopting specific strategies and using specific landmarks is crucial for successful navigation and was associated with better spatial navigation performance in typical and atypical aging but this may not apply to route-learning performance in patients with mild AD dementia. Serial position effects varied with respect to cognitive performance and type of the task. Specifically, in the route-repetition task, we found a primacy effect in both typical and atypical aging, while primacy and recency effects were present in the route-retracing task in $\mathrm{CN}$ older adults together with a recency effect in patients with aMCI and mild AD dementia. More effective spatial navigation performance was associated predominantly with better memory and executive functions. These findings indicate different aspects of spatial navigation and visuospatial strategies in typical and atypical aging that can be revealed by a realistic-looking and ecologically valid spatial navigation test suite.

Author Contributions: M.L. and J.L. participated in the design of the study, data interpretation, and wrote the draft of the manuscript. J.M.W. was involved in the design of the study and data interpretation. J.K. and V.M. were involved in spatial navigation and neuropsychological data acquisition. M.V. and J.H. were involved in data acquisition and interpretation. All authors have read and agreed to the published version of the manuscript.

Funding: This study was supported by the European Regional Development Fund-Project ENOCH no. CZ.02.1.01/0.0/0.0/16_019/0000868; the Ministry of Health, Czech Republic—conceptual development of research organization, University Hospital Motol, Prague, Czech Republic grant no. 00064203; the Institutional Support of Excellence 2. LF UK grant no. 6990332; and the Grant Agency of Charles University grant no. 327821.

Institutional Review Board Statement: The study was conducted according to the guidelines of the Declaration of Helsinki, and approved by the Ethics Committee of the Motol University Hospital and Second Faculty of Medicine, Charles University (EK-701/16, 25.5.2016).

Informed Consent Statement: Informed consent was obtained from all participants involved in the study.

Data Availability Statement: The data presented in this study are available on request from the corresponding author. The data are not publicly available due to privacy restrictions. 
Acknowledgments: The authors would like to thank M. Dokoupilova, K. Kopecka, J. Korb, S. Krejcova, H. Markova, K. Veverova, and T. Nikolai for help with data collection; J. Cerman, O. Lerch, I. Trubacik Mokrisova, Z. Nedelska, and I. Wolfova for help with participant recruitment; and M. Uller for help with MRI data processing.

Conflicts of Interest: The authors declare that the research was conducted in the absence of any commercial or financial relationships that could be construed as a potential conflict of interest.

\section{References}

1. Waller, D.; Lippa, Y. Landmarks as beacons and associative cues: Their role in route learning. Mem. Cognit. 2007, 35, 910-924. [CrossRef]

2. Muffato, V.; Meneghetti, C. Learning a Path from Real Navigation: The Advantage of Initial View, Cardinal North and VisuoSpatial Ability. Brain Sci. 2020, 10, 204. [CrossRef]

3. Hartley, T.; Maguire, E.A.; Spiers, H.J.; Burgess, N. The well-worn route and the path less traveled: Distinct neural bases of route following and wayfinding in humans. Neuron 2003, 37, 877-888. [CrossRef]

4. Maguire, E.A.; Burgess, N.; Donnett, J.G.; Frackowiak, R.S.; Frith, C.D.; O'Keefe, J. Knowing where and getting there: A human navigation network. Science 1998, 280, 921-924. [CrossRef] [PubMed]

5. Zhang, H.; Ekstrom, A. Human neural systems underlying rigid and flexible forms of allocentric spatial representation. Hum. Brain Mapp. 2013, 34, 1070-1087. [CrossRef] [PubMed]

6. Moffat, S.D. Aging and spatial navigation: What do we know and where do we go? Neuropsychol. Rev. 2009, 19, 478-489. [CrossRef] [PubMed]

7. Lester, A.W.; Moffat, S.D.; Wiener, J.M.; Barnes, C.A.; Wolbers, T. The Aging Navigational System. Neuron 2017, 95, 1019-1035. [CrossRef] [PubMed]

8. Rodgers, M.K.; Sindone, J.A.; Moffat, S.D. Effects of age on navigation strategy. Neurobiol. Aging 2012, 33, $202 . e 15-202 . e 22$. [CrossRef]

9. Goeke, C.; Kornpetpanee, S.; Köster, M.; Fernández-Revelles, A.B.; Gramann, K.; König, P. Cultural background shapes spatial reference frame proclivity. Sci. Rep. 2015, 5, 11426. [CrossRef] [PubMed]

10. Gazova, I.; Laczó, J.; Rubinova, E.; Mokrisova, I.; Hyncicova, E.; Andel, R.; Vyhnalek, M.; Sheardova, K.; Coulson, E.J.; Hort, J. Spatial navigation in young versus older adults. Front. Aging Neurosci. 2013, 5. [CrossRef] [PubMed]

11. Guderian, S.; Dzieciol, A.M.; Gadian, D.G.; Jentschke, S.; Doeller, C.F.; Burgess, N.; Mishkin, M.; Vargha-Khadem, F. Hippocampal Volume Reduction in Humans Predicts Impaired Allocentric Spatial Memory in Virtual-Reality Navigation. J. Neurosci. 2015, 35, 14123-14131. [CrossRef] [PubMed]

12. Urgolites, Z.J.; Kim, S.; Hopkins, R.O.; Squire, L.R. Map reading, navigating from maps, and the medial temporal lobe. Proc. Natl. Acad. Sci. USA 2016, 113, 14289-14293. [CrossRef]

13. Iaria, G.; Palermo, L.; Committeri, G.; Barton, J.J.S. Age differences in the formation and use of cognitive maps. Behav. Brain Res. 2009, 196, 187-191. [CrossRef]

14. Driscoll, I.; Davatzikos, C.; An, Y.; Wu, X.; Shen, D.; Kraut, M.; Resnick, S.M. Longitudinal pattern of regional brain volume change differentiates normal aging from MCI. Neurology 2009, 72, 1906. [CrossRef]

15. Braak, H.; Del Tredici, K. The preclinical phase of the pathological process underlying sporadic Alzheimer's disease. Brain 2015, 138, 2814-2833. [CrossRef]

16. Jacobs, H.I.; Van Boxtel, M.P.; Jolles, J.; Verhey, F.R.; Uylings, H.B. Parietal cortex matters in Alzheimer's disease: An overview of structural, functional and metabolic findings. Neurosci. Biobehav. Rev. 2012, 36, 297-309. [CrossRef]

17. Cushman, L.A.; Stein, K.; Duffy, C.J. Detecting navigational deficits in cognitive aging and Alzheimer disease using virtual reality. Neurology 2008, 71, 888-895. [CrossRef] [PubMed]

18. Laczó, J.; Vlcek, K.; Vyhnálek, M.; Vajnerová, O.; Ort, M.; Holmerová, I.; Tolar, M.; Andel, R.; Bojar, M.; Hort, J. Spatial navigation testing discriminates two types of amnestic mild cognitive impairment. Behav. Brain Res. 2009, 202, 252-259. [CrossRef] [PubMed]

19. Allison, S.L.; Fagan, A.M.; Morris, J.C.; Head, D. Spatial Navigation in Preclinical Alzheimer's Disease. J. Alzheimer's Dis. 2016, 52, 77-90. [CrossRef]

20. Kerbler, G.; Nedelska, Z.; Fripp, J.; Laczó, J.; Vyhnalek, M.; Lisý, J.; Hamlin, A.; Rose, S.; Hort, J.; Coulson, E. Basal Forebrain Atrophy Contributes to Allocentric Navigation Impairment in Alzheimer's Disease Patients. Front. Aging Neurosci. 2015, 7, 185. [CrossRef] [PubMed]

21. Nedelska, Z.; Andel, R.; Laczó, J.; Vlcek, K.; Horinek, D.; Lisy, J.; Sheardova, K.; Bures, J.; Hort, J. Spatial navigation impairment is proportional to right hippocampal volume. Proc. Natl. Acad. Sci. USA 2012, 109, 2590-2594. [CrossRef] [PubMed]

22. Hallab, A.; Lange, C.; Apostolova, I.; Özden, C.; Gonzalez-Escamilla, C.; Klutmann, S.; Brenner, W.; Grothe, M.J.; Buchert, R. Impairment of Everyday Spatial Navigation Abilities in Mild Cognitive Impairment Is Weakly Associated with Reduced Grey Matter Volume in the Medial Part of the Entorhinal Cortex. J. Alzheimer's Dis. 2020, 78, 1149-1159. [CrossRef] [PubMed]

23. Benke, T.; Karner, E.; Petermichl, S.; Prantner, V.; Kemmler, G. Neuropsychological deficits associated with route learning in Alzheimer disease, MCI, and normal aging. Alzheimer Dis. Assoc. Disord. 2014, 28, 162-167. [CrossRef] [PubMed]

24. Weniger, G.; Ruhleder, M.; Lange, C.; Wolf, S.; Irle, E. Egocentric and allocentric memory as assessed by virtual reality in individuals with amnestic mild cognitive impairment. Neuropsychologia 2011, 49, 518-527. [CrossRef] [PubMed] 
25. Iaria, G.; Petrides, M.; Dagher, A.; Pike, B. Cognitive strategies dependent on the hippocampus and caudate nucleus in human navigation: Variability and change with practice. J. Neurosci. 2003, 23, 5945-5952. [CrossRef]

26. Parizkova, M.; Lerch, O.; Moffat, S.D.; Andel, R.; Mazancova, A.F.; Nedelska, Z.; Vyhnalek, M.; Hort, J.; Laczó, J. The effect of Alzheimer's disease on spatial navigation strategies. Neurobiol. Aging 2018, 64, 107-115. [CrossRef]

27. Zancada-Menendez, C.; Sampedro-Piquero, P.; Lopez, L.; McNamara, T.P. Age and gender differences in spatial perspective taking. Aging Clin. Exp. Res. 2015, 28, 289-296. [CrossRef] [PubMed]

28. Marková, H.; Laczó, J.; Andel, R.; Hort, J.; Vlček, K. Perspective taking abilities in amnestic mild cognitive impairment and Alzheimer's disease. Behav. Brain Res. 2015, 281, 229-238. [CrossRef]

29. Quental, N.M.D.; Brucki, S.M.D.; Bueno, O.F.A. Visuospatial function in early Alzheimer's disease: Preliminary study. Dement. Neuropsychol. 2009, 3, 234-240. [CrossRef]

30. Faubert, J. Visual perception and aging. Can. J. Exp. Psychol. 2002, 56, 164-176. [CrossRef]

31. Ramanoël, S.; Durteste, M.; Bécu, M.; Habas, C.; Arleo, A. Differential Brain Activity in Regions Linked to Visuospatial Processing During Landmark-Based Navigation in Young and Healthy Older Adults. Front. Hum. Neurosci. 2020, 14. [CrossRef]

32. Uhlhaas, P.J.; Pantel, J.; Lanfermann, H.; Prvulovic, D.; Haenschel, C.; Maurer, K.; Linden, D.E. Visual perceptual organization deficits in Alzheimer's dementia. Dement. Geriatr. Cogn. Disord. 2008, 25, 465-475. [CrossRef]

33. Paxton, J.L.; Peavy, G.M.; Jenkins, C.; Rice, V.A.S.; Heindel, W.C.; Salmon, D.P. Deterioration of visual-perceptual organization ability in Alzheimer's disease. Cortex 2007, 43, 967-975. [CrossRef]

34. Molitor, R.J.; Ko, P.C.; Ally, B.A. Eye movements in Alzheimer's disease. J. Alzheimer's Dis. 2015, 44, 1-12. [CrossRef]

35. Kimura, K.; Reichert, J.F.; Kelly, D.M.; Moussavi, Z. Older Adults Show Less Flexible Spatial Cue Use When Navigating in a Virtual Reality Environment Compared With Younger Adults. Neurosci. Insights 2019, 14. [CrossRef]

36. Foo, P.; Warren, W.H.; Duchon, A.; Tarr, M.J. Do humans integrate routes into a cognitive map? Map- versus landmark-based navigation of novel shortcuts. J. Exp. Psychol. Learn. Mem. Cogn. 2005, 31, 195-215. [CrossRef]

37. Diersch, N.; Wolbers, T.; el Jundi, B.; Kelber, A.; Webb, B. The potential of virtual reality for spatial navigation research across the adult lifespan. J. Exp. Biol. 2019, 222. [CrossRef]

38. Wiener, J.M.; Caroll, D.; Moeller, S.; Bibi, I.; Ivanova, D.; Allen, P.; Wolbers, T. A novel virtual-reality-based route-learning test suite: Assessing the effects of cognitive aging on navigation. Behav. Res. Methods 2020, 52, 630-640. [CrossRef] [PubMed]

39. Mitrushina, M.; Satz, P.; Chervinsky, A.; D’Elia, L. Performance of four age groups of normal elderly on the Rey Auditory-Verbal Learning Test. J. Clin. Psychol. 1991, 47, 351-357. [CrossRef]

40. Kasper, E.; Brueggen, K.; Grothe, M.J.; Bruno, D.; Pomara, N.; Unterauer, E.; Duering, M.; Ewers, M.; Teipel, S.; Buerger, K. Neuronal correlates of serial position performance in amnestic mild cognitive impairment. Neuropsychology 2016, 30, 906-914. [CrossRef] [PubMed]

41. Hilton, C.; Wiener, J.; Johnson, A. Serial memory for landmarks encountered during route navigation. Q. J. Exp. Psychol. 2021. [CrossRef] [PubMed]

42. Sheardova, K.; Vyhnalek, M.; Nedelska, Z.; Laczo, J.; Andel, R.; Marciniak, R.; Cerman, J.; Lerch, O.; Hort, J. Czech Brain Aging Study (CBAS): Prospective multicentre cohort study on risk and protective factors for dementia in the Czech Republic. BMJ Open 2019, 9, e030379. [CrossRef] [PubMed]

43. Laczó, J.; Andel, R.; Vyhnalek, M.; Matoska, V.; Kaplan, V.; Nedelska, Z.; Lerch, O.; Gazova, I.; Moffat, S.D.; Hort, J. The effect of TOMM40 on spatial navigation in amnestic mild cognitive impairment. Neurobiol. Aging 2015, 36, 2024-2033. [CrossRef]

44. Parizkova, M.; Lerch, O.; Andel, R.; Kalinova, J.; Markova, H.; Vyhnalek, M.; Hort, J.; Laczó, J. Spatial Pattern Separation in Early Alzheimer's Disease. J. Alzheimer's Dis. 2020, 76, 121-138. [CrossRef]

45. McKhann, G.M.; Knopman, D.S.; Chertkow, H.; Hyman, B.T.; Jack, C.R., Jr.; Kawas, C.H.; Klunk, W.E.; Koroshetz, W.J.; Manly, J.J.; Mayeux, R.; et al. The diagnosis of dementia due to Alzheimer's disease: Recommendations from the National Institute on Aging-Alzheimer's Association workgroups on diagnostic guidelines for Alzheimer's disease. Alzheimer's Dement. 2011, 7, 263-269. [CrossRef]

46. Scheltens, P.; Leys, D.; Barkhof, F.; Huglo, D.; Weinstein, H.C.; Vermersch, P.; Kuiper, M.; Steinling, M.; Wolters, E.C.; Valk, J. Atrophy of medial temporal lobes on MRI in "probable" Alzheimer's disease and normal ageing: Diagnostic value and neuropsychological correlates. J. Neurol. Neurosurg. Psychiatry 1992, 55, 967-972. [CrossRef] [PubMed]

47. Petersen, R.C. Mild cognitive impairment as a diagnostic entity. J. Intern. Med. 2004, 256, 183-194. [CrossRef] [PubMed]

48. de Condappa, O.; Wiener, J.M. Human place and response learning: Navigation strategy selection, pupil size and gaze behavior. Psychol. Res. 2016, 80, 82-93. [CrossRef] [PubMed]

49. Wiener, J.M.; de Condappa, O.; Harris, M.A.; Wolbers, T. Maladaptive bias for extrahippocampal navigation strategies in aging humans. J. Neurosci. 2013, 33, 6012-6017. [CrossRef] [PubMed]

50. DeIpolyi, A.; Rankin, K.; Mucke, L.; Miller, B.; Gorno-Tempini, M. Spatial cognition and the human navigation network in AD and MCI. Neurology 2007, 69, 986-987. [CrossRef] [PubMed]

51. Pengas, G.; Patterson, K.; Arnold, R.L.; Bird, C.M.; Burgess, N.; Nestor, P.J. Lost and found: Bespoke memory testing for Alzheimer's disease and semantic dementia. J. Alzheimer's Dis. 2010, 21, 1347-1365. [CrossRef]

52. Cherrier, M.; Mendez, M.F.; Perryman, K. Route learning performance in Alzheimer disease patients. Neuropsychiatry. Neuropsychol. Behav. Neurol. 2001, 14, 159-168. [PubMed] 
53. Guariglia, C.C. Spatial working memory in Alzheimer's disease: A study using the Corsi block-tapping test. Dement. Neuropsychol. 2007, 1, 392-395. [CrossRef] [PubMed]

54. Wolbers, T.; Wiener, J.M. Challenges for identifying the neural mechanisms that support spatial navigation: The impact of spatial scale. Front. Hum. Neurosci. 2014, 8. [CrossRef]

55. Cornell, E.H.; Heth, C.D.; Kneubuhler, Y.; Sehgal, S. Serial Position Effects in Children's Route Reversal Errors: Implications for Police Search Operations. Appl. Cogn. Psychol. 1996, 10, 301-326. [CrossRef]

56. Helstrup, T.; Magnussen, S. The mental representation of familiar, long-distance journeys. J. Environ. Psychol. 2001, 21, 411-421. [CrossRef]

57. Weitzner, D.S.; Calamia, M. Serial position effects on list learning tasks in mild cognitive impairment and Alzheimer's disease. Neuropsychology 2020, 34, 467-478. [CrossRef]

58. Talamonti, D.; Koscik, R.; Johnson, S.; Bruno, D. Predicting Early Mild Cognitive Impairment with Free Recall: The Primacy of Primacy. Arch. Clin. Neuropsychol. 2020, 35, 133-142. [CrossRef] [PubMed]

59. Burkart, M.; Heun, R.; Benkert, O. Serial position effects in dementia of the Alzheimer type. Dement. Geriatr. Cogn. Disord. 1998, 9 , 130-136. [CrossRef] [PubMed]

60. Hort, J.; Laczó, J.; Vyhnálek, M.; Bojar, M.; Bureš, J.; Vlček, K. Spatial Navigation Deficit in Amnestic Mild Cognitive Impairment. Proc. Natl. Acad. Sci. USA 2007, 104, 4042-4047. [CrossRef]

61. Visser, P.J.; Scheltens, P.; Verhey, F.R.; Schmand, B.; Launer, J.L.; Jolles, J.; Jonker, C. Medial temporal lobe atrophy and memory dysfunction as predictors for dementia in subjects with mild cognitive impairment. J. Neurol. 1999, 246, 477-485. [CrossRef]

62. Frankenmolen, N.L.; Fasotti, L.; Kessels, R.P.C.; Oosterman, J.M. The influence of cognitive reserve and age on the use of memory strategies. Exp. Aging Res. 2018, 44,117-134. [CrossRef]

63. Moffat, S.D.; Kennedy, K.M.; Rodrigue, K.M.; Raz, N. Extrahippocampal contributions to age differences in human spatial navigation. Cereb. Cortex 2007, 17, 1274-1282. [CrossRef] [PubMed]

64. Wei, E.X.; Anson, E.R.; Resnick, S.M.; Agrawal, Y. Psychometric Tests and Spatial Navigation: Data From the Baltimore Longitudinal Study of Aging. Front. Neurol. 2020, 11. [CrossRef] [PubMed]

65. Moffat, S.D.; Zonderman, A.B.; Resnick, S.M. Age differences in spatial memory in a virtual environment navigation task. Neurobiol. Aging 2001, 22, 787-796. [CrossRef]

66. Cogné, M.; Taillade, M.; N’Kaoua, B.; Tarruella, A.; Klinger, E.; Larrue, F.; Sauzéon, H.; Joseph, P.-A.; Sorita, E. The contribution of virtual reality to the diagnosis of spatial navigation disorders and to the study of the role of navigational aids: A systematic literature review. Ann. Phys. Rehabil. Med. 2016. [CrossRef]

67. Taillade, M.; Sauzéon, H.; Dejos, M.; Pala, P.A.; Larrue, F.; Wallet, G.; Gross, C.; N'Kaoua, B. Executive and memory correlates of age-related differences in wayfinding performances using a virtual reality application. Aging Neuropsychol. Cogn. 2013, 20, 298-319. [CrossRef]

68. Laczó, J.; Andel, R.; Nedelska, Z.; Vyhnalek, M.; Vlcek, K.; Crutch, S.; Harrison, J.; Hort, J. Exploring the contribution of spatial navigation to cognitive functioning in older adults. Neurobiol. Aging 2017, 51, 67-70. [CrossRef] [PubMed]

69. Tangen, G.G.; Engedal, K.; Bergland, A.; Moger, T.A.; Hansson, O.; Mengshoel, A.M. Spatial navigation measured by the Floor Maze Test in patients with subjective cognitive impairment, mild cognitive impairment, and mild Alzheimer's disease. Int. Psychogeriatr. 2015, 27, 1401-1409. [CrossRef] 